\title{
Un territoire d'attaches : lien aux lieux et lien aux autres dans le périurbain francilien
}

\author{
Mariane Thébert ${ }^{\mathrm{a}}$, Monica Coralli ${ }^{\mathrm{b}}$, Hélène Nessic \\ et Patricia Sajous ${ }^{\mathrm{d}}$ \\ ${ }^{a}$ IFSTTAR chargée de recherche, Université Paris-Est, IFSTTAR - Laboratoire Ville Mobilité \\ Transport : 5 boulevard Copernic, Cité Descartes F 77454 Marne-la-Vallée cedex 2 \\ ${ }^{b}$ LAA / UMR LAVUE ; \\ ${ }^{c}$ UMR LAVUE Mosaïque, MCF à Nanterre Paris Ouest-la Défense ; \\ ${ }^{d}$ UMR IDEES, Université du Havre MCF
}

\section{Résumé}

Cet article traite du lien des résidents périurbains au territoire habité et des pratiques par lesquelles il se manifeste. La façon dont ce lien se construit et évolue donne à voir un processus d'ancrage spatial et social, qui se traduit par un rétrécissement des bassins de vie pour certains motifs de déplacement ainsi que par l'adoption progressive de normes comportementales valorisant la capacité à se satisfaire et à contribuer au développement des ressources parfois cachées du territoire, notamment par l'investissement associatif. Les potentialités de développement d'une « altermobilité » sont discutées au regard de ces aspirations à la proximité qui, loin de s'appréhender par la seule métrique des véhicules.kilomètres, renvoie à la territorialisation du lien social et à la transformation par les résidents du périurbain d'un contexte spatial en ressource quotidienne.

Mots clés : périurbain, mobilité, proximité, ancrage, sociabilités, covoiturage. 


\begin{abstract}
Summary
A home Territory: Ties with Places, Ties with others in the Paris suburban Area. This article discusses the individuals' relationship to the territory in the suburban areas. The way this link is built and evolves shows a spatial and social anchoring process, resulting in a narrowing of the living areas for certain trip purposes and the progressive use of behavioural norms (strong associative investment, ability to exploit «invisible» resources of the territory). The potential for development of an «other-mobility» are presented here in relation to these aspirations to the proximity that, far from being understood solely by the single measure of vehicles-per-kilometer, refers to the regionalization of the social bond and the processing by residents of the spatial context of a suburban daily resource.
\end{abstract}

Keywords: suburbs, mobility, proximity, anchoring, sociability, carpooling.

\title{
Introduction
}

Réactualisé par les interrogations sur le rapport à l'espace de "sociétés à individus mobiles » (Stock, 2001), l'intérêt des sciences sociales pour les enjeux liés à l'habiter a contribué à renouveler le regard scientifique porté sur l'espace périurbain. En contrepoint du décryptage des logiques globales (économiques et politiques) de production d'un modèle d'habitat pavillonnaire, l'attention s'est portée sur le façonnement du territoire par les pratiques habitantes. Le rapport des individus et des groupes sociaux à l'espace géographique se construit dans l'interaction non déterministe entre des formes matérielles, pour partie produites par ces forces exogènes, et les liens situés qui se tissent entre actants du territoire (Pattaroni et al., 2009 ; Corneloup et al., 2014). Relevant de ce déplacement du cadre d'analyse, les modes d'habiter décrivent ces « pratiques en situation de vie » qui produisent de l'habitabilité en construisant des liens matériels et symboliques au(x) lieu(x) (Corneloup et al., 2014 ; Cailly, 2008). Leur analyse établit des passerelles entre organisation spatiale et organisation sociale, en décrivant la façon dont le social se construit dans un territoire.

Depuis une dizaine d'années, plusieurs recherches se sont attachées à (re)caractériser un mode d'habiter périurbain, en insistant d'une part sur sa diversité et d'autre part sur la place de l'espace local dans des pratiques spatiales et des identités auxquelles était

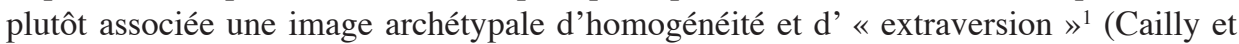
Dodier, 2007, Fourny et al., 2012, Bonnin-Oliveira et al., 2014, Berger et al., 2014, etc.). Certaines d'entre elles, ayant pour terrain d'étude l'Ile-de-France, montrent la « mutation progressive » que connait le périurbain depuis les années 1990 (Bouleau et Mettetal, 2014) et les formes de «maturité » qui le recomposent aussi bien dans ses formes, son offre, que dans les pratiques de ses habitants (Rougé, 2012). On assiste en particulier à un « ancrage » accru à l'échelle du territoire de proximité, perceptible à travers l'inflexion des pratiques de mobilité (Bouleau et Mettetal, 2014).

La matière de cet article est tirée d'une recherche collective (Aguiléra et al., 2014) qui s'inscrit dans la lignée de ces travaux. En réponse à la consultation « Du périur-

${ }^{1}$ Le périurbain étant traditionnellement décrit comme l'espace consacré aux ménages avec enfants des classes moyennes pratiquant une forme d'évasion spatiale permanente. 
bain à l'urbain » organisée par le Plan Urbanisme Construction Architecture, elle s'est attachée à décrypter, par la mobilisation des méthodes qualitative et quantitative, l'évolution sur le temps long des modes d'habiter et des territoires périurbains au travers des pratiques de mobilité résidentielle et quotidienne. Les terrains sont situés dans le périurbain francilien, à environ trente kilomètres au nord, à l'est et au sud-ouest de Paris. Les enseignements retirés de l'exploitation d'une quarantaine d'entretiens semidirectifs auprès de ménages, d'élus et de responsables associatifs ont été mis en regard avec quelques éléments quantifiés extraits d'un questionnaire passé auprès des résidents des mêmes terrains.

Les résultats présentés ici relèvent de l'investigation qualitative du lien au territoire, dans ses composantes géographiques et sociales : comment se construit-il et évolue-t-il ? Quelles sont les pratiques situées dans lesquelles il se forge et à travers lesquelles il se manifeste ? Quel rapport à la proximité sociale et spatiale se noue dans l'espace périurbain ? Bien que les modes d'habiter s'appréhendent aussi en dehors de la quotidienneté (Stock, 2001), c'est pour leur récurrence que les pratiques ici analysées ont été prises en considération, en tant qu'elles construisent la capacité à trouver au quotidien les ressources nécessaires au maintien de soi, dans une perspective tant écologique (Mathieu, 2012) qu'anthropologique (Di Méo, 1996 ; Breviglieri, 2009). Ainsi l'espace local se réimpose à l'analyse comme «base essentielle de la vie quotidienne », y compris dans le contexte de forte mobilité (Charmes, 2008). Il s'impose dans la matérialité de périmètres spatiaux qu'il faut délimiter, comme dans le processus plus idéel d'appropriation du territoire qui relève de l'échelle individuelle mais aussi de la construction collective de sens et de l'usage partagé des lieux.

L'installation dans le périurbain, bien que dépendante de logiques macro-économiques, suscite un enracinement dans le territoire : la première partie de l'article expose les méthodes, les terrains et l'analyse des trajectoires résidentielles sur lesquels se fonde ce constat. À partir du domicile, l'espace local se trouve progressivement investi. En deuxième partie, l'étude de la territorialisation des liens sociaux et du phénomène associatif est indispensable pour comprendre selon quelles modalités se fait cette installation dans le périurbain : c'est par ce biais que les ménages se donnent les moyens de la maîtrise d'un environnement qui demande apprentissage, et transforment un espace résidentiel choisi pour ses qualités génériques en un espace élu, pourvoyeur de repères et support d'identification. C'est aussi par ce biais qu'ils ancrent dans l'espace local une part non négligeable de leurs activités : en troisième partie, l'analyse des mobilités quotidiennes permet de préciser quels sont les périmètres spatiaux de l'ancrage, mais aussi les modes de fréquentation du territoire local qui signalent la façon dont les ménages vivent et éprouvent au quotidien la proximité.

Ces analyses se focalisent sur les pratiques socio-spatiales communes aux différents entretiens. Celles-ci sont apparues prépondérantes par rapport aux différenciations induites par les caractéristiques sociales et économiques des individus. Il ne s'agit nullement de nier l'influence de ces paramètres, démontrée par ailleurs et à l'origine de typologies de profils très fines (Dodier, 2007a ; Sencébé et Hilal, 2012 ; etc.), mais plutôt d'apprécier certains aspects de «l'effet de lissage » qui caractérise le périurbain francilien, et renvoie bien à la maturité d'un tissu ancien dans lequel les effets de l'ancrage sont sensibles (Sencébé et Hilal, 2012). 
Qu'il s'agisse de jouir des aménités « urbaines » locales ou des ingrédients sociaux du « village », les modalités périurbaines de construction du rapport à la proximité sociale et spatiale et la façon dont cet entre-deux ville-village devient au quotidien une ressource pour ceux qui y résident permettent d'expliciter les raisons pour lesquelles ils aiment y vivre.

\section{Saisir les dynamiques périurbaines en Ile-de-France : méthode, terrains et parcours résidentiels des populations}

Les territoires étudiés, distants d'une trentaine de kilomètres de Paris, ont été touchés par la «vague » périurbaine dès la première moitié des années 1970, avec le maintien d'une très forte croissance sur toute la décennie, un tassement relatif durant les années 1980 et, sur certains communes, un redémarrage dans les années 1990 (Conti, 2012). Leur population a doublé en quarante ans et ils continuent aujourd'hui d'attirer de nouveaux résidents. Différentes « générations » d'arrivants périurbains y cohabitent. Le développement d'une approche compréhensive de la relation qu'ils établissent avec leur territoire de résidence commence par l'analyse des motifs qui les ont conduits à s'installer aux marges de l'agglomération, et surtout des facteurs qui les poussent à rester.

Pour saisir la diversité des parcours, nous avons croisé les méthodes quantitative et qualitative, et privilégié à chaque étape d'enquête une approche longitudinale visant à saisir l'évolution parallèle des territoires et des modes d'habiter. Ces éléments concernant les trajectoires et leurs liens avec les contextes résidentiels coïncident avec les constats effectués sur des terrains variés (Aragau et al., 2012 ; Fortin et Després, 2008 ; Authier et al., 2010). Ils permettent également d'établir que les terrains étudiés sont loin d'appartenir au périurbain de relégation.

\subsection{Appréhender l'évolution des territoires, des mobilités et des modes d'habiter sur trois territoires représentatifs de la grande couronne francilienne}

Le choix des terrains, trois communautés de communes franciliennes, s'explique par l'objectif général de la recherche dans laquelle s'inscrit cet article : étudier les dynamiques périurbaines au travers des mobilités et analyser les enjeux en matière de politique de transport. Ces choix n'ont donc pas été effectués en référence à la thématique du lien social traitée dans cet article, qui s'est imposée au cours des investigations. Les territoires étudiés, situés à distance équivalente de Paris, s'insèrent dans des contextes métropolitains un peu différents mais n'ont pas été directement touchés par des projets d'aménagement urbain ou de transport majeurs. Si des investissements lourds ont eu lieu à proximité, les mutations qui les ont affectés sont indépendantes d'un volontarisme politique d'échelle nationale ou régionale.

À l'est, un premier terrain est constitué des cinq communes composant l'intercommunalité de la Brie Boisée, qui accueillent toutes moins de 2000 habitants. Elles se situent en bordure sud de la ville nouvelle de Marne-la-Vallée (secteur Val d'Europe et Chessy), à proximité du RER A et de l'autoroute A4.

Au nord, la communauté de communes de Carnelle - Pays de France regroupe dix communes et 20967 habitants. Contrairement aux deux autres, ce territoire n'est pas situé à proximité immédiate d'une ville nouvelle. Au sud de l'EPCI, la N104 offre un accès rapide aux bassins d'emplois de Cergy et Roissy. Une ligne de Transilien (ligne H) parcourt le territoire et conduit jusqu'à la Gare du Nord. 
Au sud-ouest, les neuf communes de la Haute Vallée de Chevreuse constituent le troisième terrain, marqué par la proximité de la ville nouvelle de Saint-Quentin-en-Yvelines et la présence historique d'une ligne de chemin de fer (désormais intégrée au RER B), dont le terminus est situé sur la communauté de communes. Malgré l'absence de grands axes routiers structurants, il s'agit d'une des zones où l'utilisation des transports collectifs est la plus faible à distance égale de Paris, ce qui peut en partie s'expliquer par la composition sociale du territoire.

Carte 1 : Des terrains représentatifs du contexte francilien en matière de revenus par ménage. Source : IAU idf- INSEE 2013, auteur : H. Nessi.

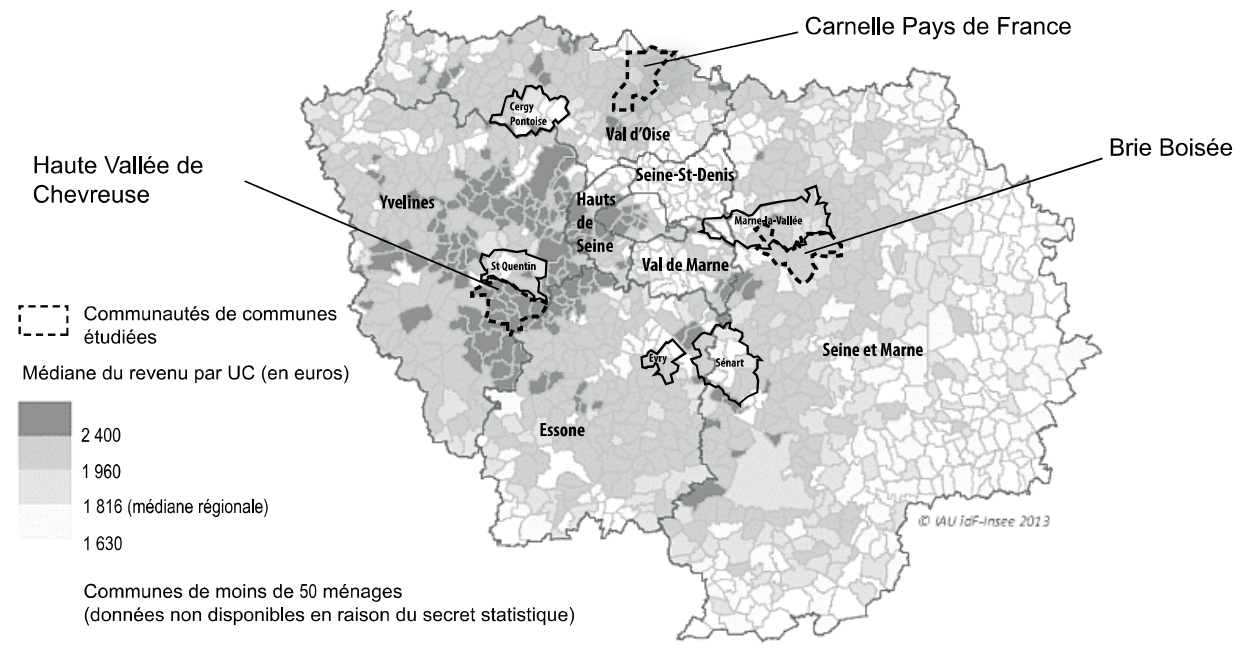

En effet, la Haute Vallée de Chevreuse se distingue par les niveaux de revenu et de diplôme très élevés de sa population. Les deux autres terrains se situent dans la fourchette de revenus qui caractérise les communes situées à cette distance de Paris (carte 1), et la distribution de leurs résidents selon la catégorie de diplômes est plutôt moins favorable que celle d'échelle régionale ${ }^{2}$. On trouve en Haute vallée de Chevreuse deux fois plus de cadres et professions intellectuelles supérieures qu'à la Brie Boisée et à Carnelle - Pays de France. Ces deux terrains, dominés par les « groupes de type moyen » selon la typologie socio-spatiale de Preteceille (2003), abritent toujours une majorité de professions intermédiaires et d'employés (Conti, 2012). Si les recompositions résidentielles restent à l'œuvre puisque les trois terrains continuent d'accueillir de nouveaux résidents, elles se nourrissent désormais d'une plus grande variété de flux migratoires. La logique unidirectionnelle de desserrement depuis le centre ne suffit plus à expliquer le sens préférentiel des trajectoires résidentielles.

\footnotetext{
${ }^{2}$ En 2008, le pourcentage de diplômés universitaires (27 \% à la Brie Boisée, $26 \%$ à Carnelle - Pays de France) est deux fois moindre qu'en Haute Vallée de Chevreuse (49\%) et un peu en dessous des $35 \%$ de l'Ilede-France. Ces deux terrains se distinguent surtout par la surreprésentation des diplômés d'un CAP ou BEP (respectivement $56 \%$ et $23 \%$, contre $17 \%$ à l'échelle régionale) (Conti, 2012).
} 
Grâce aux premières enquêtes numérisées (Recensement Général de Population, Enquête Globale de Transport), une étude quantitative a retracé l'évolution sociodémographique des terrains et des mobilités domicile-travail sur quarante ans (Conti, 2012). Une enquête par questionnaire ${ }^{3}$ portant sur le parcours résidentiel et la mobilité quotidienne tous motifs a ensuite été réalisée pour obtenir davantage de données précisément localisées sur nos terrains. L'échantillon de réponses est représentatif de la structure locale des populations (tableau 1), que ce soit pour le nombre de personnes par ménage, le type de logement, l'âge ou le statut socio-professionnel ${ }^{4}$.

\begin{tabular}{|c|c|c|c|c|c|c|c|}
\hline Age & $\begin{array}{c}\text { Territoires } \\
\text { d'étude }\end{array}$ & Questionnaire & $\begin{array}{c}\text { Entretiens } \\
\text { qualitatifs }\end{array}$ & $\begin{array}{c}\text { Statut } \\
\text { professionnel }\end{array}$ & $\begin{array}{c}\text { Territoires } \\
\text { d'étude }\end{array}$ & Questionnaire & $\begin{array}{c}\text { Entretiens } \\
\text { qualitatifs }\end{array}$ \\
\hline$<30$ ans & $6 \%$ & $6 \%$ & $6 \%$ & $\begin{array}{c}\text { Inactifs / } \\
\text { Chômeurs }\end{array}$ & $4 \%$ & $5 \%$ & $7 \%$ \\
\hline $31-50$ ans & $40 \%$ & $45 \%$ & $45 \%$ & Etudiants & $0 \%$ & $0 \%$ & $0 \%$ \\
\hline $51-64$ ans & $32 \%$ & $27 \%$ & $26 \%$ & Retraités & $28 \%$ & $29 \%$ & $23 \%$ \\
\hline$>65$ ans & $22 \%$ & $22 \%$ & $23 \%$ & Actifs & $66 \%$ & $64 \%$ & $70 \%$ \\
\hline
\end{tabular}

Tableau 1 : Quelques caractéristiques sociodémographiques des territoires d'étude (moyennes réalisées à partir du RGP 2010, INSEE) et des populations enquêtées.

Enfin, une enquête qualitative a approfondi la connaissance biographique des parcours périurbains, dont la diversité ne se laisse plus enfermer dans le schéma de desserrement depuis le centre métropolitain décrit par les analystes du phénomène périurbain pour les années 1970-1980. Trente-et-un entretiens semi-directifs en face-à-face ont été conduits auprès de ménages ayant presque tous répondu au questionnaire. Les mobilités résidentielle et quotidienne ont ainsi été replacées dans leur contexte historique, à la fois personnel et métropolitain (relocalisation des emplois, commerces et services à l'échelle locale et dans l'ensemble de la métropole). Cette démarche a permis, par exemple, de saisir les reconfigurations affectant les schémas quotidiens de déplacement au gré de la transformation des terrains (cf. partie 3), et d'affirmer que le renforcement de la place de l'espace local dans les modes d'habiter périurbains ne tient pas au seul renouvellement du regard porté sur ces espaces. La grille d'entretien comportait aussi un champ axé sur la mobilisation du local (réseau social et aménités territoriales) comme ressource, et sur la gestion de la mobilité au sein des foyers en tant qu'outil essentiel de maîtrise du quotidien. L'étude de la dynamique associative a été complétée par douze entretiens auprès d'élus et de responsables associatifs, et par la collecte d'informations à l'occasion des rencontres annuelles que sont les forums des associations.

${ }^{3} 1800$ questionnaires ont été distribués entre avril et juin 2013 dans les boîtes aux lettres de quatre communes sur chacun des trois terrains, en veillant à diversifier le profil des communes en matière de démographie, de densité et de niveaux d'équipement. Le taux de retour (317 réponses exploitables) est supérieur à $15 \%$.

${ }^{4}$ On relève une légère surreprésentation des 31-50 ans au détriment des 51-64 ans (écart = 5\%), et des locataires (écart $=8 \%$ ). 
L'échantillon des personnes rencontrées comporte plus de femmes que d'hommes, et les familles nombreuses y sont surreprésentées ${ }^{5}$. On y trouve néanmoins des ménages de petite taille et des familles monoparentales. La structure par âge et la répartition par CSP ne présentent pas de distorsion notable par rapport à la structure locale des populations (tableau 1). Les populations rencontrées dans le cadre de nos enquêtes quantitative et qualitative reflètent la diversification des profils périurbains et l'atypisme moins marqué de ces territoires par rapport à l'agglomération (Aragau et al., 2012). Ces trois terrains sont également représentatifs de la couronne francilienne dans laquelle ils s'insèrent. Cela signifie qu'ils n'offrent pas à l'analyse l'ensemble du spectre social (surtout dans ses composantes défavorisées), mais permettent d'apprécier l'influence sur les pratiques d'un « effet de localisation » plus prononcé que dans d'autres types d'espaces (Sencébé et Hilal, 2012).

\subsection{Pourquoi venir? Le sens des trajectoires résidentielles}

L'examen des précédents lieux de résidence des habitants qui, en 2008, déclaraient habiter une autre commune cinq ans auparavant révèle une évolution significative des logiques migratoires. Elles se font majoritairement au sein du même département (Conti, 2012). Pour la majorité des personnes rencontrées et pour plus des deux-tiers des répondants au questionnaire, le dernier déménagement s'est effectué au sein de la grande couronne, correspondant à une translation graduelle de périurbain à périurbain un peu plus lointain. Le passage de l'agglomération à la ville diffuse est progressif et multi-étapes. Il a parfois été effectué par la génération précédente. Mais l'arrivée dans le périurbain est présentée comme l'aboutissement «naturel » de ces parcours résidentiels diversifiés, et la commune de résidence représente, pour les ménages rencontrés, davantage qu'une réserve de foncier accessible choisie par défaut.

$\mathrm{Si}$ on retrouve, dans l'énoncé des critères de leur choix résidentiel, les déterminants classiques de la dynamique de desserrement (famille agrandie, désir d'accession à la propriété en maison individuelle), ceux-ci se combinent étroitement avec la mise en exergue des ressources propres au territoire : proximité d'un emploi, de la famille et, systématiquement, des aménités associées à la campagne (verdure, calme, tranquillité...). L'éloignement de l'agglomération est donc aussi un rapprochement, et la diversité des motifs à l'origine du choix de localisation souligne la diversification fonctionnelle du périurbain. La structure du marché immobilier, évidemment contraignante, a exercé une détermination qui parait surtout locale : le schéma dominant présente l'arrivée dans la commune de résidence comme résultant d'un « hasard », tandis que le secteur a fait

${ }^{5}$ L'échantillon est composé de 8 hommes et 23 femmes. La surreprésentation des ménages de plus de quatre personnes y est de $18 \%$. On note là aussi une légère surreprésentation des 31-50 ans au détriment des 51-64 ans (écart $=5 \%$ ) et des plus de 60 ans ( $1 \%$ ). En termes de CSP, l'échantillon est composé de 5 cadres et professions intellectuelles supérieures, 10 professions intermédiaires, 4 employés, 9 retraités, 1 chômeur, 2 personnes sans activité professionnelle. Les inactifs/chômeurs et les actifs (écart de 2 et $3 \%$ ) sont légèrement surreprésentés au détriment des retraités $(-4 \%)$. 
l'objet d'un choix. La contrainte des coûts influence certes le degré d'excentrement ${ }^{6}$, mais il reste que, au-delà des critères (prioritaires) liés au logement même, les ménages recherchent un cadre de vie tout simplement incompatible avec le bâti aggloméré.

Indissociables de l'habitabilité périurbaine, les dimensions de la ruralité ${ }^{7}$ fondent la lecture rétrospective du parcours résidentiel telle que livrée dans les entretiens, faisant du périurbain une sorte de biotope vers lequel inclinent « naturellement » ses résidents, le territoire d'évidence pour réaliser son projet de vie familiale. Les discours font ainsi régulièrement état d'une familiarité avec la campagne acquise au cours de l'enfance. L'attrait pour le vert constitue le fil directeur qui donne sa cohérence à la variété des espaces habités. Les parcours résidentiels sont en effet riches et diversifiés (seuls $8 \%$ des répondants au questionnaire n'ont jamais déménagé), avec des échappées lointaines sur le territoire national ou à l'étranger. L'urbain très dense n'y est pas un passage obligé. Mais plusieurs enquêtés inscrivent la majeure partie de leurs pérégrinations résidentielles à l'intérieur de la grande couronne francilienne, dans un secteur mordant parfois sur la petite couronne et au sein duquel ils ont expérimenté différents degrés de densité. C'est souvent à cette échelle de secteur que se déploie le territoire de référence identitaire. Cette dynamique alimente la connaissance du territoire périurbain : l'opposition ville-campagne, si elle reste structurante des représentations, se dilue au profit d'une gamme plus graduée de territoires, associant la campagne, le bourg, la petite ville, la commune voisine en pleine expansion... et procurant des ressources variées et abondantes. Ce système de lieux n'évince pas l'agglomération principale des territoires fréquentés, mais il l'exclut du champ des possibles résidentiels. Si le retour dans l'agglomération n'est pas l'horizon virtuel des couples qui se défont (Dodier, 2007a), qui vieillissent (Aragau et Morel-Brochet, 2010) ou dont les enfants grandissent ${ }^{8}$, c'est que le territoire résidentiel comble un appétit auto-proclamé pour la ruralité qui va bien au-delà de la jouissance d'un jardin, sans impliquer pour autant un renoncement aux aménités urbaines nécessaires pour se projeter dans le long terme.

\subsection{Pourquoi rester? Un processus d'assimilation territoriale durable}

Les personnes rencontrées ne font que très rarement état d'un projet de déménagement à court ou moyen terme. Seuls $25 \%$ des répondants au questionnaire l'envisagent, pour des raisons mécaniques (logement devenu inadapté ou changement professionnel) qui n'incriminent que très minoritairement le territoire habité et ses ressources. S'il arrive que le départ soit envisagé à long terme, toute relocalisation se fera(it) à caractéristiques « rurales » constantes, voire accentuées, « loin du bruit, ça c'est sûr » et « encore plus dans la nature ». Lorsque le projet est plus concret, ancré dans un futur moins lointain ou même dans un passé récent, un critère prédomine : le maintien d'un réseau social fortement territorialisé.

\footnotetext{
${ }^{6}$ D'abord au sein du secteur via les caractéristiques de raccordement aux réseaux de transport individuel et collectif de la commune économiquement éligible, mais probablement aussi en amont, les ménages intégrant les contraintes de l'offre dans le bornage du secteur de recherche (Dodier, 2007a).

${ }^{7}$ Perçues comme telles et qui vont de la qualité de l'air à une forme d'atmosphère sociale, en passant bien sûr par les caractéristiques paysagères.

${ }^{8}$ Sans en faire un motif de relocalisation, quelques mères de famille relèvent toutefois que, s'il y a « tout sur place » pour les enfants en bas âge, les choses se compliquent à l'entrée au collège.
} 
"[Après le divorce] Je suis restée ici parce que des voisins gentils, charmants (...)

Il y a beaucoup de gens qui restent les mêmes (...). Donc on se connaît très bien. » (Josée, Brie Boisée, Villeneuve-le-Comte, 82 ans, veuve, retraitée)

"Je voulais rester dans le coin, parce qu'en fin de compte, ben, les amis sont là. »

(Christine, Carnelle - Pays de France, Viarmes, 64 ans, veuve, retraitée)

Divorce et veuvage ont pu motiver un déménagement dans une maison plus petite ou vers le bourg le plus proche, mais dans une logique du moindre éloignement qui met à profit une offre de logements légèrement diversifiée ${ }^{9}$. Il ressort de ces cas rencontrés que l'espace périurbain est un espace « à maturité » (Berger et al., 2014), capable d'absorber les aléas de l'existence et les recompositions de la cellule familiale. La dissolution de celle-ci est insuffisante à fonder systématiquement des velléités de retour en zone beaucoup plus dense, même dans le cas d'un parcours résidentiel ayant fait la part belle à l'agglomération. L'habiter périurbain s'avère porteur d'une logique assurantielle qui dépasse le logement : même en situation de précarité économique ou d'isolement lié à l'incapacité de conduire, le territoire habité reste une ressource en ce qu'il offre, d'une part, un toit dont la possible perte n'est jamais évoquée ; d'autre part, du lien social permettant de mutualiser les biens et les compétences.

Ainsi, au décès de son mari, Amina (Haute Vallée de Chevreuse, Mesnil-Saint-Denis, 45 ans, veuve, aide-soignante) a-t-elle conservé la voiture du ménage alors qu'elle ne conduit pas : la voiture conserve une valeur d'usage grâce à quelques ami(e)s qui peuvent l'utiliser tout en véhiculant sa propriétaire. Ivan (Haute Vallée de Chevreuse, Dampierre-en-Yvelines, célibataire, élagueur, invalide) a "un copain qui est venu habiter chez [lui] pour [l']aider». "Il a une voiture, moi non, il n'avait plus de logement, et moi oui. Cet hiver, quand la voiture de mon copain a eu un problème, c'est un autre qui est venu nous chercher après son boulot $»$. Le maintien dans le territoire, même dans les conditions particulièrement difficiles que génère la dépendance automobile, est la condition du maintien de soi.

Enfin, et dans des conditions économiques et sociales très différentes, un départ envisagé à long terme ne signifie pas rupture des liens avec un territoire qui est devenu, dans les représentations, le lieu où l'on rentre chez soi, l'élément stable dans des parcours résidentiels riches en mobilité, le lieu où les petits enfants, nés et à venir, un parent en difficulté, les amis même lointains peuvent être accueillis, selon les besoins et sur des temporalités variables.

"On garde la maison quoiqu'il arrive [même si départ pour d'autres horizons]. Ça sert de référence aussi pour les enfants. Ils savent qu'on peut revenir là. C'est psychologique. » (Agathe, Brie Boisée, Ferrières-en-Brie, 48 ans, mariée, femme au foyer) "Je voulais une maison avec une grande pièce... J'ai cinqpetits-enfants. Bon, à l'époque, je n'en avais pas tout à fait autant... Mais je voulais pouvoir les recevoir, sinon ils ne viennent pas. » (Christine, Carnelle - Pays de France, Viarmes, 64 ans, veuve, retraitée)

L'analyse des mobilités résidentielles passées ou projetées montre que l'installation dans le périurbain procède d'un choix de long terme. Se démarquant des espaces centraux où le renouvellement des populations est perçu par les enquêtés comme étant plus rapide, le périurbain offre une certaine stabilité, liée notamment à la propriété d'une maison suffisamment vaste pour répondre sur le long terme aux besoins d'une cellule familiale

${ }^{9}$ Le diagnostic du Scot de la Brie Boisée constate par exemple le doublement sur le territoire de la part des logements collectifs et de l'offre locative entre 1990 et 1999. 
à géométrie mouvante. Le périurbain est ainsi le lieu d'une «permanence territoriale » (Aragau et al., 2011), affective et matérielle. Erigé en patrimoine hérité et transmis, il constitue un repère stable sur le temps long, celui de plusieurs générations, et impose un sens à la complexité des parcours résidentiels en les faisant converger vers un territoire porteur d'identité. Il est le lieu où l'on revient, et les quelques rêveries d'évasion s'incarnent d'ailleurs dans des espaces qui en conservent les caractéristiques. Même dans les cas de situation économique difficile (voire de réelle précarité), le lien avec le territoire reste positif parce qu'il repose soit sur un fondement identitaire et affectif fort, soit sur une appréciation très favorable des évolutions de celui-ci ${ }^{10}$.

Bien que contingente dans le concept de mode d'habiter (Stock, 2001), la dimension du temps long est en revanche au cœur de l'habitabilité périurbaine. À rebours de l'archétype du parcours résidentiel périurbain, et contrairement à certains quartiers agglomérés ${ }^{11}$, le périurbain n'est pas un espace de transit consacré à une étape interstitielle du projet de vie : c'est un espace d'enracinement résidentiel. C'est aussi un espace d'ancrage, c'est-àdire de liens établis avec le territoire qui s'éprouvent et se pratiquent à l'échelle du quotidien et de ses mobilités. Conséquence de la diversification des parcours résidentiels, les ménages rencontrés ne sont que rarement des néo-ruraux susceptibles d'être déstabilisés par un choix dont ils n'auraient pas mesuré les implications en termes de mode de vie, et nous verrons en partie 3 qu'ils s'estiment majoritairement à portée de toutes les ressources nécessaires. Néanmoins, cette satisfaction n'est pas donnée d'emblée. C'est à la faveur de la pérennité de leur installation que les ménages périurbains vont développer de véritables savoir-faire territoriaux, fondés sur l'appropriation progressive des ressources environnantes à la fois sociales et fonctionnelles.

\section{L'ancrage comme processus social : des savoir-faire en action}

C'est l'ancrage en tant que processus qui est analysé ici, en reconstituant l'établissement progressif de liens sociaux inscrits dans l'espace et qui vont médiatiser la relation avec le territoire. La force de ces liens faibles (de Coninck, 2010) s'apprécie dans la façon dont ils viennent en relais du système automobile, mais participent aussi de la fabrication d'un véritable savoirfaire territorial. Cette analyse de la construction d'un espace « du proche » permet de comprendre comment un contexte spatial, souvent présenté comme contraignant, se transforme en ressource pour ses habitants qui y composent leur propre assemblage spatio-temporel.

\subsection{Le déploiement spatial et temporel des différents cercles de la familiarité}

L'enjeu des pratiques sociales et spatiales qui manifestent un ou des modes d'habiter est d'établir un usage familier de l'environnement (Breviglieri, 2009). Le lien social dessine

\footnotetext{
${ }^{10}$ Cette affirmation doit être nuancée sur le terrain de Carnelle - Pays de France où quelques enquêtés expriment le sentiment d'une dégradation de l'offre territoriale (commerces et desserte en transport) : l'âge plus élevé et l'ancienneté de la présence sur le territoire semblent faire la différence.

${ }^{11}$ Notamment, les espaces péricentraux « refondés », résultant de grandes opérations immobilières de rénovation, accueillent des résidents dont le départ à court ou moyen terme est planifié dans une large majorité des cas, ce qui influence considérablement le lien au local (Cousin, 2014).
} 
une territorialité à la fois aréolaire, par l'inscription dans l'espace immédiat de la co-habitation (Haumont, 2005), et réticulaire, par le biais de l'appartenance à différents groupes qui introduisent de la distance dans la logique de contiguïté spatiale. Ce processus requiert du temps, dans l'absolu (quelques années) mais aussi du temps de présence locale : ce «temps résidentiel » n'est pas réductible à la « résidentialité » dédiée à la sphère domestique et à un mode de vie casanier (Hilal et Sencébé, 2002). Il conduit à investir l'espace extérieur au domicile (d'autant plus volontiers que la localisation résidentielle donne satisfaction) et permet la création, entre résidents, d'un lien de connaissance d'abord visuel. Il est dégagé à la faveur d'une mise entre parenthèses des activités professionnelles ou d'horaires de travail décalés, atypiques, partiels ou simplement très flexibles, généralisés dans notre échantillon et qui réactualisent l'image d'un périurbain vidé puis rempli au rythme de migrations pendulaires synchrones (Dodier, 2007b).

\subsubsection{Le voisinage : des interactions sous contrôle}

Le premier espace de familiarité qu'est le voisinage ${ }^{12}$ se caractérise par l'association de la proximité physique (et la récurrence de l'interaction qui lui est liée) avec la maîtrise du degré de proximité sociale. De l'ordre de la rencontre, les fréquentations de voisinage sont associées à l'improvisation et à un désir d'informel, en opposition à la vie relationnelle dans les grandes agglomérations.

«Les amis parisiens, vous n'allez pas les voir sans les avoir prévenus. (...). Il faut téléphoner, il faut s'organiser. La vie parisienne n'est pas la même. Vous n'allez pas sonner chez les gens comme ça.» (Christine, Carnelle - Pays de France, Viarmes, 64 ans, veuve, retraitée)

Par la récurrence de la rencontre non programmée, le voisin est un inconnu qui devient familier ; mais il devient plus rarement un ami. La relation est contenue par des codes qui la cantonnent à la brièveté et aux « espaces intermédiaires » que sont le jardin, l'impasse du lotissement (Dubost, 1984 ; Charmes, 2005 ; Nessi, 2012). Les invitations organisées chez les uns et les autres sont plutôt de l'ordre de l'exceptionnel.

"On se fait un petit coucou comme ça (...). Un petit bonjour, si ça va, une histoire d'un quart d'heure et puis je rentre chez moi. » (Maryse, Carnelle-Pays de France, St Martin-du-Tertre, 62 ans, mariée, infirmière)

«Il y a une bonne cohésion. (...). Mais simplement des relations comme cela, de maison à maison, mais sans invitation, vous voyez, on se rend visite comme ça, on passe un petit moment ensemble et puis voila. » (Joëlle, Brie Boisée, Villeneuve-le-Comte, 68 ans, mariée, retraitée)

Les relations plus personnalisées, potentiellement intrusives, pourraient menacer une cohabitation qui impose des rencontres rapides mais fréquentes. Tant qu'elle ne met pas en situation de rendre des comptes sur ce qui est offert au regard du voisin, la co-surveillance ${ }^{13}$ nourrit le sentiment de tranquillité :

\footnotetext{
${ }^{12}$ Entendu comme le même ensemble résidentiel ou le même village lorsque celui-ci est suffisamment petit pour générer un lien d'interconnaissance au moins visuelle et fondé sur le partage d'un territoire familier.

${ }^{13}$ Le « coup d'œil » jeté sur la maison du voisin durant l'absence de celui-ci étant un grand classique, illustratif d'une surveillance qui se veut purement pratique, dirigée sur l'espace partagé et non sur les individus : le voisin n'a pas officiellement annoncé qu'il s'en va, il n'a pas à expliquer où il va ni quand il rentre... mais on sait qu'il n'est pas là.
} 
«Et puis au niveau du... comment... du civisme, de tout, c'est beaucoup plus... Les gens se connaissent. Ce n'est pas... voilà... Sans se connaître, quoi...» (Catherine, Brie Boisée, Pontcarré, 55 ans, veuve/remariée, employée de la fonction publique) "On connait le voisinage proche. Enfin... Bonjour, au-revoir, on s'entend hyper bien, on surveille les maisons quand on n'est pas là, mais ça s'arrête là. » (Gabrielle, Carnelle-Pays de France, Baillet-en-France, 34 ans, mariée, RH)

L'équilibre, délicat, semble s'établir avec le temps. Les arrivants les plus récents fuient parfois ostensiblement le voisinage pour établir des liens au-delà de cette première aire géographique. En revanche, le discours des résidents plus anciens est nettement plus positif. Une fois les codes assimilés (convivialité et distance entretenue), la relation de voisinage présente des avantages appréciables : car, avec le temps, ces « liens faibles » qui ne sont pas dénués de « force » (Granovetter, 1976) déploient leurs effets bénéfiques potentiels. C'est d'abord une garantie diffuse d'assistance mutuelle face aux aléas de la vie qui est assurée par l'intégration dans le groupe, sans impliquer d'interconnaissance individuelle approfondie :

"Nos enfants jouent ensemble, bon, si... On sait que si on a un souci... même si on ne se voit pas plus que ça... On sait qu'on est là les uns pour les autres. » (Anne, Brie Boisée, Villeneuve-le-Comte, 42 ans, mariée, femme au foyer)

Ce n'est pas à des affinités électives, mais bien au caractère d'espace réduit et partagé avec quelques autres que les enquêtés renvoient systématiquement pour expliquer les interrelations. La notion d' " ambiance », souvent mobilisée, renvoie à un contexte à la fois immanent, car lié à la forme urbaine faiblement dense, et conjoncturel, c'est-à-dire susceptible de changer si les personnes changent ${ }^{14}$.

"Il y a vraiment une âme de village. Bussy ça fait vraiment cité dortoir, donc contrairement à ce que j'avais vécu là-bas où je ne voyais pas les gens, je ne voyais pas mes voisins, ici vraiment les gens se parlent, les voisins sortent...»(Cécile, Brie Boisée, Ferrière-en-Brie, 42 ans, mariée, hôtesse de l'air)

En effet, le système d'échanges qui se met en place a des effets pratiques directement fondés sur la proximité spatiale : on ne se parle pas beaucoup mais on se rend service en tondant une pelouse mitoyenne, en gardant les enfants quelques minutes, les animaux domestiques quelques jours, en réceptionnant les livraisons... Les enquêtés récusent les termes de système d'entraide en référence à ces pratiques, car la systématisation en est exclue. Mais elles participent bien de la mise en œuvre d'un "régime de proximité » (Cailly, 2014), vecteur de relations de solidarité soigneusement régulées mais denses, qui fondent un véritable « contrat de valorisation résidentielle » (ibid.).

\subsubsection{De la proximité spatiale à la constitution de groupes structurés : une étape}

indispensable à l'intégration des nouveaux arrivants

Les affinités plus électives se structurent par l'appartenance à des groupes qui prennent une certaine distance par rapport à la logique de la contiguïté spatiale et introduisent souvent la médiation d'une structure souple (associative). Regroupés autour d'un intérêt commun, les individus s'agrègent pour partager une activité ou un engagement. Les liens établis dans ces cadres institutionnalisés ne sont plus nécessairement tissés avec les

${ }^{14} \mathrm{~L}$ ' « instabilité » de quelques rares ménages, arrivés pour repartir quelques années après, est d'ailleurs assez mal vue et assimilée à une forme d'inconséquence. 
voisins immédiats, mais avec des habitants éloignés dans le village ou résidant dans les communes alentours. Ils sont souvent présentés comme plus approfondis que ceux fondés sur l'immédiate proximité ${ }^{15}$, et résistent mieux dans les cas de déménagement suivi d'un retour dans les années suivantes. L'institution opère comme un filtre qui permet de s'impliquer sans risquer de perdre en autonomie.

"[On a tissé des liens ici], pas beaucoup, mais avec les contacts de l'école, puis le sport, mon mari fait du foot, il a rencontré des copains, on se fait des barbecues, on se refait un petit monde ici (...). En gros, on a sympathisé avec plein de gens, bonjour au revoir, mais après, des gens avec qui on partage, deux. » (Carole, Haute Vallée de Chevreuse, Dampierre, 45 ans, mariée, décoratrice d'intérieur)

Ce passage par les «structures » (qui finissent par constituer du réseau, contrairement à la seule proximité) est indispensable à l'intégration. Les associations sont d'importants " pourvoyeurs » de liens sociaux, tissés autant grâce à l'assiduité des rencontres générées par l'activité que par la multiplication des moments de convivialité organisés autour de l'activité.

"[Je me suis intégrée] Parce qu'après, je me suis investie au niveau associatif. » (Anne, Brie Boisée, Villeneuve-le-Comte, 42 ans, mariée, femme au foyer)

"Quand je suis arrivée toute seule et avec mes filles, c'est en allant aux associations que j'ai fait connaissance de gens et que je me suis fait un cercle d'amis et de connaissances. » (Marie-Christine, Brie Boisée, Pontcarré, 45 ans, mariée, secrétaire)

Les enfants poussent à la fréquentation de ces structures. L'exploitation du questionnaire montre que la proportion de répondants déclarant participer à la vie locale de leur commune varie en fonction du nombre moyen d'enfants (tableau 2) ainsi que de l'âge de ceux-ci, avec un «pic » d'implication entre 10 et 15 ans. Les parents d'enfants de cette tranche d'âge ne sont que $14 \%$ à estimer qu'ils ne participent pas du tout, contre $41 \%$ pour les parents d'enfants de plus de 15 ans.

Q33 : êtes-vous d'accord avec l'affirmation suivante : " je participe à la vie de ma commune »?

\begin{tabular}{|l|c|c|c|c|}
\hline & $\begin{array}{c}\text { Tout à fait } \\
\text { d'accord }\end{array}$ & Plutôt d'accord & $\begin{array}{c}\text { Plutôt pas } \\
\text { d'accord }\end{array}$ & $\begin{array}{c}\text { Pas du tout } \\
\text { d'accord }\end{array}$ \\
\hline Nb moyen d'enfants & 1.6 & 1.4 & 1 & 0.7 \\
\hline Au moins 1 enfant & $19 \%$ & $35 \%$ & $25 \%$ & $22 \%$ \\
\hline Pas d'enfant & $12 \%$ & $25 \%$ & $26 \%$ & $37 \%$ \\
\hline
\end{tabular}

Tableau 2 : Une participation accrue à la vie locale lorsque le nombre d'enfants croit.

Tous les discours convergent sur ce point : quand les enfants ont grandi, il faut faire un effort pour maintenir le lien. Le maintien du contact passe par la participation plus ponctuelle aux manifestations de la vie locale (y compris pour aider à organiser celles qui concernent au premier chef les enfants), ou par l'adhésion aux associations qui s'adressent à un public en

${ }^{15}$ Ciblés et construits, ils permettent de s'extraire d'un déterminisme spatial qui peut avoir une connotation aliénante, voire isolante pour le nouvel arrivé. Ils permettent de revendiquer une dimension choisie et, partant, une certaine rareté liée à l'élitisme du processus. Ce trait est caractéristique des CSP favorisées qui privilégient les sociabilités électives et planifiées aux sociabilités d'immédiate proximité (Sencébé et Hilal, 2012). L'intensité de la sociabilité de voisinage reste néanmoins distinctive des espaces périurbains par rapport à l'urbain (ibid.). 
moyenne plus âgé et proposent des activités artistiques (sculpture, peinture sur soie...), de valorisation-protection du patrimoine, ou encore de randonnée.

"Une fois qu'on n'a plus d'enfants d'âge scolaire dans le secteur, ben, c'est déjà un peu plus difficile d'avoir des liens avec les habitants (...). Donc... et bon...en participant, comme ça...en allant à des réunions par les associations, ça permet de garder un petit lien, quand même, avec des gens de la commune.» (Amina, Haute Vallée de Chevreuse, Mesnil-St-Denis, 45 ans, veuve, aide-soignante)

"J'ai plein de copines qui n'ont plus d'enfant à l'école élémentaire mais qui continuent à justement faire des Trocs et Puces pour pouvoir rencontrer des gens et dire "ah, tiens...! », c'est vrai que c'est l'occasion de se retrouver (...) Moi, je ne fais pas partie d'associations, en revanche, quand on me demande un coup de main, si je suis là, je le fais. C'est important parce que c'est quand même retrouver des Ferriérois, et puis des gens qu'on a côtoyés quand les enfants étaient à l'école... des retrouvailles.» (Agathe, Brie Boisée, Ferrières-en-Brie, 48 ans, mariée, femme au foyer)

Au cours du cycle de vie, conserver sa place dans le(s) groupe(s) suscite la mise en œuvre de stratégies évolutives. Cet effort de maintien dans le réseau est indispensable : les groupes constitués jouent un rôle essentiel dans le partage (des sociabilités, d'une voiture...). Par exemple, la pratique des activités de loisirs les plus caractéristiques du milieu périurbain, comme la randonnée en forêt ou la course, s'effectue fréquemment dans le cadre associatif, alors même qu'elle ne nécessite pas d'infrastructure mutualisée. Les groupes ont une réelle importance dans le développement des pratiques d'entraide, plus soutenu dans le cadre de regroupements fondés sur une communauté d'intérêt et une base affinitaire (même ténue ${ }^{16}$ ) que sur la proximité physique.

«Nous, ici... -moi, je suis ici depuis dix ans-on s'entraide. On s'entraide parce qu'on fait partie de différents réseaux : soit l'association de parents d'élèves, soit l'AMAP... » (Entretien élu Haute Vallée de Chevreuse).

«Alors, [l'entraide] ce ne sera pas voisin-voisin, ce sera plus amis plus éloignés dans le village. » (Julie, Haute Vallée de Chevreuse, Chevreuse, 40 ans, mariée, psychologue)

Plus largement, c'est tout l'espace du collectif qui est structuré par ces appartenances : les espaces ouverts, les manifestations événementielles de la vie locale sont des lieux de pratiques partagées où se mêlent différents profils, mais ils se fréquentent par groupes de connaissance établis (y compris par le biais du voisinage). En forêt ou dans les fêtes de village, les « tribus » (famille, voisins, membres d'une association) coexistent et s'offrent un moment de «vivre ensemble », mais la rencontre y est pré-balisée ${ }^{17}$.

\footnotetext{
${ }^{16}$ La plupart des associations tendent à fédérer des publics homogènes, notamment en termes de place dans le cycle de vie. Le coût d'entrée dans le réseau peut même être facteur de tri : la participation à certaines associations est chère, moins du fait du montant de l'adhésion que par le biais des obligations qui sont faites aux adhérents en matière de renouvellement/entretien du matériel.

${ }^{17}$ En cela, on peut discuter leur assimilation aux lieux de rencontre et d'interactions plus ouvertes que constitue (raient) les espaces publics urbains. Sans trancher, on peut constater que les associations ont acquis, sur ces terrains favorisés comme dans les espaces périurbains à dominante populaire (Girard, 2014), une fonction sociale qui tend à les substituer aux lieux de rencontre des cafés d'antan : le partage de nourriture et de boissons, lors de rencontres organisées en marge des activités fondatrices, est une dimension essentielle de leur fonctionnement, qu'on retrouve au cœur de nombre des échanges à l'occasion des traditionnels forums des associations tenus annuellement dans chaque commune.
} 
"Même si ne suis pas toujours à la sortie de l'école, on arrive quand même à se voir avec les parents, et on arrive à se reconnaître dans les manifestations justement organisées par la mairie plutôt que par l'école. Bon, en fait, on arrive toujours à trouver quelqu'un qu'on connaît et c'est appréciable et c'est ça que j'aime, (...), alors mon mari fait du ping-pong et là aussi, quand on fait des brocantes, il arrive toujours à connaître quelqu'un qui fait partie aussi du club de ping-pong. » (Cécile, Brie Boisée, Ferrières-en-Brie, 42 ans, mariée, hôtesse de l'air)

Cependant, les manifestations « ouvertes », du type brocantes ou fêtes, n'en sont pas moins vécues comme des moments particuliers et décisifs pour la vie locale : si l'appartenance à un groupe est un tremplin indispensable pour y participer, elles constituent des lieux où les différentes sphères d'appartenance peuvent se recouper, une forme de percolation peut s'opérer, bref, où les groupes peuvent s'agréger, se mettre en réseau pour gagner en force.

«Avec mon mari, on boycotte les soirées «voisins», «piscine», etc. (...) La soirée piscine, on sait que ce n'est pas une soirée ouverte à tout le monde. (...), mais la «soirée saucisse-merguez-frites» au village, déjà oui, là on s'en fout de savoir combien on gagne, où on travaille, c'est compteurs à zéro. " (Carole, Haute Vallée de Chevreuse, Dampierre, 45 ans, mariée, décoratrice d'intérieur)

En effet, si les solidarités et l'espace des sociabilités obéissent à différentes segmentations $^{18}$, la multi-appartenance et le maillage des réseaux font qu'on n'est pas assigné à un territoire, un groupe, un profil social.

"Il y a aussi beaucoup d'associations, souvent les gens appartiennent à plusieurs en même temps. » (Mireille, Brie Boisée, Ferrières-en-Brie, 45 ans, mariée, cadre)

"Les anciens s'entraident entre eux parce qu'ils se connaissent depuis longtemps, mais par rapport aux nouveaux qui arrivent, il y en a beaucoup, ils ne connaissent pas trop (...). Par contre, où il peut y avoir des rencontres, c'est quand l'été, sur la place, ben, pour la fête de la musique, la fête de la ferme (...). Dès qu'il y a un événement qui se passe sur la place, effectivement, là, il y a du lien qui se fait, parce que toutes les générations viennent et se retrouvent. » (Anne, Brie Boisée, Villeneuve-leComte, 42 ans, mariée, femme au foyer)

"Il y a différents petits réseaux comme ça, qui sont bien ancrés dans le territoire et c'est grâce à ces réseaux que derrière... tout se maille en fait. Les personnes qui arrivent et n'ont pas les réseaux restent seules. » (Entretien élu Haute Vallée de Chevreuse)

L'appartenance à différents groupes inscrit l'individu dans un réseau ramifié qui l'introduit dans un collectif territorialisé sans être aliénant.

\subsubsection{De l'associatif au collectif : la constitution d'un «nous »}

La richesse de l'offre associative, systématiquement mise en avant dans les discours, participe du processus d'identification territoriale en générant, à partir des engagements individuels, de l'identité collective locale. C'est le tissu des relations instaurées par le biais de ces structures qui permet l'animation et la mise en scène du territoire à travers les manifestations festives. La «vie locale» périurbaine relève ainsi d'une imbrication particulière entre responsabilité individuelle et responsabilité collective.

${ }^{18}$ Appartenance au même ensemble résidentiel, à la même famille, à la même école, à la même association, au même socio-type : jeunes actifs avec enfants, retraités, parents d'adolescents, etc. 
Si l'implication individuelle est souvent minorée dans un premier temps du discours, cette assertion ne résiste pas au détail des faits : les entretiens qualitatifs permettent de relativiser l'exploitation du questionnaire, par le biais duquel un habitant sur deux estime ne pas ou peu participer à la vie locale ${ }^{19}$. Beaucoup d'enquêtés, seuls ou « avec quelques copains et copines », ont ainsi participé activement à l'animation locale, investissant des « vides » autant créateurs de nécessités que générateurs de possibles.

"Si la gymnastique a débuté, c'est parce que c'est moi qui l'ai fait débuter. Et je l'ai fait débuter parce qu'on avait des enfants dans les jambes. " (Josée, Brie Boisée, Villeneuve-le-Comte, 82 ans, veuve, retraitée)

"J'ai repris le club de foot pendant quatre mois parce qu'ils n'avaient pas de président, mais je l'ai fait plus parce que c'était des gamins qui avaient besoin de continuer à jouer au foot. » (Carole, Haute Vallée de Chevreuse, Dampierre, 45 ans, mariée, décoratrice d'intérieur)

Le maillage trop lâche de l'offre publique laisse l'initiative se déployer depuis « la base ». Se crée une forme de culture locale de l'investissement, qui opère comme marqueur de l'intégration au groupe tout en générant une certaine pression sociale. Il faut savoir saisir le relais tendu par les autres, et l'attitude qui consiste à « consommer » des services associatifs est dénoncée comme étant le fait des tout nouveaux arrivants, étrangers à la communauté. Même lorsque la puissance publique locale prend le relais, les individus conservent une part importante de responsabilité, un sens de l'obligation qui exprime la puissance du social s'imposant à des individus qui l'ont intériorisé (Baudin, Bonnin, 2009).

«La Mairie organise énormément de choses, donc moi j’essaie de participer, quand on voit qu'il y a un effort... on essaie d'y être, autrement c'est vrai, si on n'est pas là, ils feront de moins en moins de choses. » (Cécile, Brie Boisée, Ferrières-en-Brie, 42 ans, mariée, hôtesse de l'air)

Cette responsabilité collective est souvent présentée comme caractéristique des petites communautés. Elle implique une forme de mobilisation permanente, et effective même en dehors de statut officiel : c'est l'individu qui apporte sa compétence mais aussi ses contraintes propres (qu'il peut faire valoir à tout moment).

"[La vie locale] Elle me convient parce que c'est une petite commune, et que... oui, je trouve qu'une association qui monte un gala, et qui demande aux papas de bien vouloir aider à monter les décors, les mamans à venir une soirée au lieu de regarder tout... Vous voyez... au lieu d'être spectateur un soir, je vais aller aider les gamines à se maquiller... Oui, je trouve ça sympa. Voilà. C'est la vie qu'on a envie de mener. » (Stéphanie, Carnelle - Pays de France, Viarmes, 34 ans, mariée, commerciale)

«Moi, je veux bien donner des cours, (...). À la limite, je me sens tout à fait capable. (...) Ce qu'il y a, c'est que je ne veux pas être coincée tous les jours... ou une fois par semaine à heure fixe parce que ça... (...). J'ai dit : il faut qu'il y ait un peu de liberté. Ils m'ont dit : oui, il n'y a pas de soucis. »(Christine, Carnelle - Pays de France, Viarmes, 64 ans, veuve, retraitée)

\footnotetext{
${ }^{19}$ On peut supposer ici l'effet de l'intériorisation d'une norme exigeante en la matière, tirée par la figure du « surinvesti » telle que décrite par Dodier (2007a).
} 
Cette représentation de la «petite communauté » induit une relation particulière à la puissance publique locale. Trop lui demander est un comportement déplacé, de nouvel arrivant. La frontière entre institution et individu est perméable, et la première ne se substitue jamais totalement au second.

"L'intérêt de garder des petits bassins de vie, c'est que la proximité c'est essentiel.

Même pour la solidarité. » (Entretien élu Haute Vallée de Chevreuse)

Bien sûr, le renouvellement de la population induit une pression en faveur du développement des équipements locaux dont les effets sont connus. Quant à la valorisation anglosaxonne et idéologique du neighborhood, elle ne correspond pas à la réalité périurbaine française. Mais celle-ci se démarque néanmoins par le développement de pratiques autoorganisées, dont le degré de formalisation varie tout en demeurant globalement réduit afin de rester compatible avec la flexibilité revendiquée du mode de vie.

\subsection{Liens sociaux et accès aux ressources}

L'entraide se pratique entre voisins, ainsi qu'au sein de groupes d'individus plus dispersés spatialement mais dont le profil s'homogénéise autour d'un trait commun (cf. point précédent). Dans ce cadre, elle gagne en structuration. En instaurant une proximité faite de règles et de codes rassurants, les groupes permettent d'envisager par exemple la pratique du covoiturage.

Beaucoup des pratiques d'entraide et d'auto-organisation sont en effet orientées autour de l'accès au système automobile, et assurent la pérennité de ce dernier plutôt que sa transformation. La dépendance du mode de vie périurbain à l'égard de l'automobile requiert la mise en œuvre de solidarités collectives pour assurer la survie de ceux qui en sont exclus, de façon temporaire ou permanente.

"Or, ici, c'est... comme je vous dis, on est quand même tributaire beaucoup de la voiture, on est quand même obligé de se serrer un peu les coudes, quoi, parce que sinon, effectivement, ça peut devenir un peu... pénible, quoi. » (Anne, Brie Boisée, Villeneuve-le-Comte, 42 ans, mariée, femme au foyer)

Ces situations de crise sont mobilisatrices mais l'aide apportée est caractérisée soit par son irrégularité, soit par sa faible fréquence, soit par le fait qu'elle s'inscrit sur une période de temps limitée. Elle est soumise aux stratégies et contraintes que l'aidant met en place ou subit dans sa propre expérience du système automobile.

"C'est arrivé très ponctuellement au début, quand on est rentré, je n'avais pas de voiture et... très ponctuellement, j'ai des copines qui m'emmenaient faire les courses quand elles allaient les faire, mais sinon, non. » (Marie, Haute Vallée de Chevreuse, Lévy-St-Nom, 37 ans, mariée, assistante maternelle)

Néanmoins, le postulat très fréquent de la réciprocité («il/elle en ferait autant») témoigne du fait qu'il s'agit bien de s'assurer une place dans la communauté en s'inscrivant dans un système de don et contre-don, même virtuel. Ce système opère bien à cette échelle communautaire et pas nécessairement à celle de la relation bilatérale instaurée à l'occasion du service rendu : ce n'est pas nécessairement la personne aidée qui rendra service en retour, spécifiquement dans le cas de l'aide apportée à une personne âgée. L'aide sera apportée le moment venu par d'autres éléments du groupe, dans le cadre d'une solidarité relativement structurée puisque les individus se relayent dès qu'il s'agit d'actes réitérés. 
"Par exemple, la personne que vous avez vue qui est invalide, tout le monde lui a demandé : tu nous dis si tu as besoin de nous ; mais comme il y a 25 personnes qui lui ont demandé, beh, enfin, jusqu'à présent, elle n'a pas fait appel à nous. » (JeanMichel, Carnelle - Pays de France, Seugy, 77 ans, marié, retraité)

En dehors de ces situations ponctuelles de mobilisation, la mobilité présente des formes auto-organisées dont la durée de vie se calque sur l'année scolaire, en premier lieu avec le développement de solutions collectives d'accompagnement des enfants.

"Chaque début d'année... bon, on organisait, il y avait... admettons, 2 ou 3 voitures qui, le matin, embarquaient tous les enfants. C'était souvent les papas qui faisaient le matin et les mamans qui faisaient le soir. En fait, ça dépendait, mais ça fonctionnait bien. » (Béatrice, Haute Vallée de Chevreuse, Dampierre, 60 ans, mariée, retraitée)

Mais le covoiturage se pratique également entre adultes en capacité de conduire et ayant un véhicule à disposition. Ce sont les activités de loisirs pratiquées en groupe qui en sont à l'origine. Les modalités (lieu de rendez-vous, voiture utilisée, horaire) se calent également sur l'année. Pour ce motif, un certain degré de systématisme dans l'organisation est accepté, car l'engagement dans la routine signe l'appartenance au groupe.

«On a des cours de conversation en anglais. Donc, on ... on y va en voiture (...) On est trois de Viarmes à y aller. Donc, on prend une voiture, à tour de rôle...» (Christine, Carnelle - Pays de France, Viarmes, 64 ans, veuve, retraitée)

"L'année dernière, je faisais de l'aquagym (...). Donc là, par contre, j'étais avec des voisines, on était sept dans la voiture...du village, et donc... c'est moi qui emmenais et qui ramenais, et c'était tous les mercredis. " (Anne, Brie Boisée, Villeneuve-leComte, 42 ans, mariée, femme au foyer)

"Le samedi soir, on essaie d'aller au cinéma, (...), on prend une seule voiture, soit des copines du village, soit une amie qui était à Chevreuse. » (Elisabeth, Haute Vallée de Chevreuse, Cernay, 48 ans, mariée, sage-femme)

Au final, le partage de la mobilité s'effectue selon des modalités et dans des limites qui ne se comprennent qu'au regard des modes d'habiter :

1. Ces pratiques sont restreintes à des cercles préalablement définis et réduits, construits autour d'une communauté de problème, d'affinités ou de caractéristiques ${ }^{20}$.

2. Elles sont réservées à certains motifs de déplacement, au premier rang desquels les loisirs et la santé, et sont quasi exclues des motifs contraints du type achats et travail ${ }^{21}$, pour lesquels les personnes se refusent à « ajouter de la contrainte à la contrainte ». À l'évocation d'un covoiturage pour motif « achats », il est courant de déclarer qu'on préfère « faire les courses pour » plutôt que convoyer.

"C'est compliqué [le covoiturage].(...). Les fois où je vais faire les courses, ce n'est pas organisé à l'avance. » (Bernard, Carnelle-Pays de France, Baillet-en-France, 65 ans, marié, retraité)

«De toute façon, on ne sait pas à quelle heure on part, on ne sait pas à quelle heure on revient. On ne sait pas combien de temps on va mettre. Et franchement, faire des

${ }^{20}$ Une proximité d'âge, une arrivée au même moment, une même position dans le cycle familial sont des facteurs de mise en contact.

${ }^{21}$ Le covoiturage domicile-travail n'est pas existant mais rare : les trois cas recensés ne sont plus d'actualité. 
détours le matin ou le soir, juste pour faire du covoiturage... Non, ça va aller. On galère déjà assez comme ça. » (Stéphanie, Carnelle - Pays de France, Viarmes, 34 ans, mariée, commerciale)

Les pratiques de cueillette ou d'achat à la ferme constituent des exceptions notables : plus rares, elles recèlent aussi une dimension ludique qui les apparente au loisir et pousse au partage. Par ailleurs, le « hasard » peut bien faire les choses, et pousser au covoiturage spontané y compris pour un motif contraint. La pratique est alors acceptée parce qu'elle est exempte de toute planification.

"La cueillette, on l'a fait, on a même rigolé, parce que là, pour le coup, j’y suis allée en covoiturage et avec un voisin, ah, ah! Et on a pris des tomates, mais... on en a eu pour... pour un régiment, quoi! » (Anne, Brie Boisée, Villeneuve-le-Comte, 42 ans, mariée, femme au foyer)

"Ça m'arrive avec mes voisines, si on voit qu'on part toutes en même temps [faire les courses], c'est déjà arrivé qu'on prenne une seule voiture, surtout le week-end.» (Mireille, Brie Boisée, Ferrières-en-Brie, 45 ans, mariée, cadre)

3. Elles ne doivent pas être systématiques : à l'exception de certains loisirs, nous n'observons pas sur nos terrains la mise en place des routines de covoiturage entre adultes qu'ont pu analyser Pradel et al. (2014) sur d'autres terrains. S'il existe bien des « formes récurrentes d'arrangements », qui contribuent au maintien du système social et spatial de l'habiter périurbain, elles ne se perpétuent qu'à condition d'échapper à une trop forte rigidité programmatique, malgré la tension périurbaine des programmes d'activité et la dispersion des lieux. Le savoir-faire automobile (Sajous, 2004) est mobilisé pour fluidifier les routines et non en instaurer. Sur nos terrains (franciliens, plutôt privilégiés), le répertoire des possibles ( «éseaux », plans $\mathrm{B}$, contre-don à faire valoir...) est un répertoire non routinier, de crise exclusivement.

"Ça m'est arrivé de co-voiturer, mais c'est moi qui ramenais des personnes quand elles avaient des problèmes de voiture, mais mon mari et moi, on a chacun une voiture, pour l'instant je ne co-voiture pas mais j'ai un réseau dans le coin, au cas où ça puisse arriver. » (Elisabeth, Haute Vallée de Chevreuse, Cernay, 48 ans, mariée, sage-femme)

$\mathrm{Au}$ vu de ces limites, c'est bien l'auto-stop, pratique la plus adaptée à cette double revendication d'organisation des solidarités territoriales et de préservation de l'incertitude, qui fait figure d'institution. Les entretiens témoignent qu'il s'agit d'un mode de faire répandu sur les trois terrains, et dont le succès repose sur la combinaison de l'absence de programmation et de l'interconnaissance.

«Pratiquement... pas tous les jours mais...je prenais des gens de Pontcarré pour les emmener au RER. [C'était des gens que vous connaissiez déjà ?] On se connaissait... en parlant. » (Marie-Christine, Brie Boisée, Pontcarré, 45 ans, mariée, secrétaire)

"Quand j'amène mes enfants à l'école, ça m'arrive de croiser des parents d'élèves qui me proposent de m'amener au RER plutôt que d'attendre le bus, parce qu'ils y vont ...» (Mireille, Brie Boisée, Ferrières-en-Brie, 45 ans, mariée, cadre)

Cette interconnaissance peut être visuelle (on connaît de vue tous les habitants de la commune) mais aussi se fonder sur une forme de «caution territoriale » délivrée par le fait qu'il est «normal » de faire de l'auto-stop sur tel trajet (par exemple celui qui consiste à aller vers le RER en l'absence de transports collectifs assurant un rabattement jugé correct). 
Sur nos terrains, l'auto-organisation porte sur l'échange de services plus que sur le troc de produits. Ce fait caractérise un tissu social aisé, mais aussi un tissu périurbain dans lequel la gestion des tensions entre distance et proximité nécessite de jouer simultanément d'un savoir-faire automobile et d'un savoir-faire territorial. Or, ce savoir-faire territorial ne consiste plus à exploiter les produits de la terre, mais à développer des solutions auto-organisées pour éviter des déplacements, plutôt que celles (majoritaires) destinées à maintenir les habitants dans la mobilité22.

« [le club de gymnastique], je l'ai fait débuter (...) parce qu'il n'y avait rien... On ne pouvait pas toujours prendre la voiture. » (Josée, Brie boisée Villeneuve-le-Comte, 82 ans, veuve, retraitée)

La prise en charge mutualisée des enfants est un grand classique de l'auto-organisation périurbaine, dont l'objectif est bien d'éviter des véhicules.km. Le degré d'organisation est ici important, avec une répartition précisément arrêtée de qui fait quoi et quand, en fonction de ce que ça coûte à chacun en distance.

"Le midi, en fait, on prenait les enfants, avec trois mamans, on s'était arrangées, on prenait chacune notre tour les enfants. Et donc, moi, je devais les avoir le lundi, l'autre maman le mardi, une autre le jeudi, et moi, je reprenais le vendredi, mais c'était une fois sur deux avec la deuxième maman. La troisième ne pouvait pas parce que c'est elle qui habitait le plus loin, si vous voulez... » (Anne, Brie Boisée, Villeneuve-le-Comte, 42 ans, mariée, femme au foyer)

Ce savoir-faire territorial se traduit plus généralement par la capacité à décrypter toutes les potentialités du territoire. Cette assimilation territoriale passe par l'intégration sociale.

\subsection{Liens sociaux et apprentissage du territoire}

Cette société auto-organisée ne se donne pas à lire d'emblée. Se faire une place sur le territoire est relativement long. Tout d'abord, les normes comportementales qui régissent les relations de voisinage comme les groupes s'acquièrent progressivement. Or le tissage de ces liens conditionne l'intégration dans le collectif élargi. En conséquence, le sentiment de participer à la vie locale s'affirme avec le temps. Le seuil des trois ans après l'emménagement semble peu compressible ${ }^{23}$. Seule l'insertion dans les réseaux permet de faire l'apprentissage d'un territoire périurbain dont les ressources doivent être décodées.

"Quand on arrive, ou quand on est extérieur, on a l'impression qu'il n'y a pas grand-chose... (...). Moi, ça ne m'attirait pas particulièrement, honnêtement. Mais en fait, quand on y vit, on s'aperçoit qu'il y a plein... plein de petites choses... que ce soit la petite pièce de théâtre locale, que ce soit le petit concert, que ce soit la brocante machin...» (Eléonore, Haute Vallée de Chevreuse, Dampierre-en-Yvelines, 34 ans, mariée, informaticienne)

Beaucoup d'activités et d'animations fonctionnent sur un mode événementiel qui n'engendre aucune consolidation dans le bâti. Leur inscription territoriale est éphémère

\footnotetext{
${ }^{22} \mathrm{Y}$ compris en gardant les enfants pour faciliter le déplacement du parent.

${ }^{23} \mathrm{Si}$ en moyenne $49 \%$ des répondants au questionnaire estiment participer à la vie communale, ils sont seulement un peu plus de $20 \%$ lorsqu'ils habitent la commune depuis moins de 3 ans, $47 \%$ si l'ancienneté est comprise entre 3 et 5 ans, et $53 \%$ au-delà.
} 
ou invisible. L'offre de commerces et services actuellement en développement n'a pas « pignon sur rue », s'effectuant souvent à domicile pour les services à la personne ou à la ferme pour la vente de produits alimentaires. Elle se développe par le bouche à oreille au sein des différents réseaux d'interconnaissance (voisinage, école, associations, paroisse). L'existence de circuits de consommation des produits issus d'exploitations agricoles proches est attestée sur les trois terrains, pour des denrées variées. Si l'achat se fait au lieu de production, il faut en connaître les modalités, parfois complexes, voire discrètes.

"On a une ferme, mais là, il faut téléphoner, ce n'est pas une boutique, si vous voulez, il faut passer un coup de fil, ils vous disent tel jour, de telle heure à telle heure. » (Anne, Brie Boisée, Villeneuve-le-Comte, 42 ans, mariée, femme au foyer) «Ça [l'AMAP], je l'ai trouvé un peu... un peu par hasard...donc, j'en fais profiter...mon amie...vvoilà. »(Christine, Carnelle - Pays de France, Viarmes, 64 ans, veuve, retraitée) "Il y a eu à [commune voisine] un fermier qui a tenu boutique, jusqu'à il y a huit ou dix ans, après (...) les normes européennes ont fait que il a été obligé de fermer boutique et il n'a plus le droit d'en vendre, sauf au noir apparemment mais il faut aller à sa ferme...» (Jean-Michel, Carnelle - Pays de France, Seugy, 77 ans, marié, retraité) Parfois, la commande est passée puis réceptionnée par le biais d'un système auto-organisé entre voisins, le réseau social de voisinage permettant le repérage et l'exploitation des opportunités locales de consommation.

«En fait, c'est une maman qui est à l'école, et on lui commande, et elle nous livre (...) Ma voisine, tout ce qu'elle commande, c'est déposé chez moi et puis après, on lui remet, quoi. Elle passe par moi pour que je passe commande à la maman, que je vois à l'école, pour ses légumes et tout, parce que c'est pas la même école. » (Anne, Brie Boisée, Villeneuve-le-Comte, 42 ans, mariée, femme au foyer)

Cet apprentissage des potentialités du territoire est une dimension du processus d'assimilation territoriale. Le changement de discours est net entre les personnes arrivées depuis quelques années (moins de cinq) et les personnes installées depuis plus longtemps. Quand les seconds s'estiment (de plus en plus) à proximité de tout, les premiers, sans remettre en question leur choix de localisation, font parfois état de la rareté des ressources locales et du manque d'activités les poussant hors du domicile en dehors du travail. Mais chez ces individus, le processus d'ancrage s'esquisse d'ores et déjà dans la participation (encore timide) aux événements locaux dont la variété, progressivement découverte, constitue une agréable surprise. Les quelques critiques concernant l'absence locale de services s'effacent rapidement chez les nouveaux arrivants au profit d'une acceptation et d'une exploitation approfondie des ressources de proximité. Les anciens reconnaissent qu' « au début, c'était difficile».

L'ancrage consiste en l'inscription de l'individu dans un réseau. Inséré dans ce réseau, l'individu n'est plus assigné à son territoire de résidence : il « habite » un secteur plus large, plus réticulé, riche d'opportunités. Ce réseau est à la fois social (et c'est la condition pour rompre un isolement potentiel que la seule fréquentation «par hasard » des voisins ne suffit pas à rompre) et spatial : le réseau des territoires « vécus » et fréquentés au quotidien, qui se déploie lui aussi à une échelle qui dépasse l'espace de résidence tout en s'articulant fortement autour de lui. 


\section{Les périmètres spatiaux de l'ancrage : une pratique quotidienne du local}

Les ménages rencontrés ont le sentiment d'avoir trouvé un compromis maîtrisé et durable entre urbanité et ruralité. Le sentiment de vivre à la campagne, partagé par $88 \%$ des répondants au questionnaire, se combine ainsi avec une large satisfaction (71\%) quant à l'accès aux emplois, commerces et services du quotidien. Ce compromis périurbain repose sur les ressources offertes par les pôles qui maillent la grande couronne francilienne (Bonnin-Oliveira et al., 2014). La reconstitution des schémas quotidiens de déplacement permet d'apprécier la place de l'espace local dans cet archipel des lieux fréquentés. De fait, une part non négligeable des programmes d'activités s'effectue dans un périmètre qu'on peut qualifier, comme le font les ménages, de proche, à condition de préciser les échelles spatiales que recouvre ce terme. Ce périmètre de proximité est investi de plus en plus fortement : parce que ses ressources sont exploitées plus assidûment à mesure que se déploie le processus d'assimilation territoriale évoqué précédemment ; mais aussi parce qu'elles s'y trouvent en nombre croissant et que les modalités de fréquentation du local répondent aux aspirations des ménages en matière de choix et de flexibilité. Animé d'échanges croisés, ce bassin d'ancrage s'avère complexe à structurer dans une perspective d'action publique.

\section{1. «Ici, nous avons tout ce qu'il nous faut! » : les deux échelles de l'ancrage}

Tous les discours expriment une représentation semblable du territoire habité : ils dessinent une territorialité d'abord aréolaire, investissant les abords immédiats d'un domicile confiné au cœur d'espaces verts qui sont le support de nombreuses pratiques habitantes ; puis se déployant sur le mode réticulaire dans un «système de lieux » (Pinson, Thomann, 2002) qui englobe, proche mais toujours en lisière, la « ville », que référence soit ici faite aux polarités d'une vingtaine de milliers d'habitants dispersées dans un rayon d'une quinzaine de kilomètres, ou à l'agglomération parisienne.

"C'est une qualité de vie, car on n'est quand même pas loin de la ville, tout en étant en dehors de la ville. On est entouré de forêts. » (Claude, Haute Vallée de Chevreuse, Chevreuse, 65 ans, veuve, retraitée)

Cette organisation duale ville/campagne doit rester immuable : $70 \%$ des répondants au questionnaire se déclarent défavorables à l'accueil de population supplémentaire sur leur commune, même dans la perspective d'accueillir en parallèle davantage de commerces et services. La dépendance automobile est acceptée car elle permet ce maintien à distance de l'activité urbaine. La priorité reste le maintien d'une ambiance « de village ». Si l'évolution du territoire est majoritairement perçue comme très positive par les enquêtés, c'est qu'elle associe le maintien d'un statu quo très localisé et le renforcement de l'armature des aménités disponibles alentours.

"Sinon, ce village, il a quelque chose de très bien, c'est qu'à mes yeux, il est très statique. Il n'évolue pas. » (Béatrice, Haute Vallée de Chevreuse, Dampierre, 60 ans, mariée, retraitée)

«Le maire a fait tout ce qu'il pouvait pour conserver un petit peu cette option de village, avec des commerces de proximité, parce qu'on a absolument tout dans le village pour vivre. » (Marie-Christine, Brie Boisée, Pontcarré, 45 ans, mariée, secrétaire) 
"S'il y avait plus, ça gâcherait le côté petit village (...); il y a assez de centres commerciaux aux alentours, on n'a pas besoin de ça ici. Ça ne me dérange pas de prendre la voiture.» (Cécile, Brie Boisée, Ferrières-en-Brie, 42 ans, mariée, hôtesse de l'air)

La pratique quotidienne du local renvoie donc à une double échelle, associant le très proche et un système de lieux plus éloignés. Elle fait écho à la territorialisation du lien social observée en deuxième partie. À travers les déplacements réalisés, l'ancrage se manifeste d'abord dans la force polarisante du très proche, compris dans un rayon inférieur à cinq kilomètres autour du domicile. La commune de résidence, pour peu qu'elle offre quelques commerces et services, notamment médicaux, est fréquentée avec régularité pour (par ordre d'importance de la commune de résidence dans l'activité) la santé, les activités de loisirs, les visites familiales et amicales et les courses (souvent d'appoint). Grâce aux nombreuses associations, facteur d'attractivité de la commune de résidence mentionné juste après les espaces verts, les enquêtés pratiquent des activités sportives, artistiques et aussi culturelles (concerts dans l'église, expositions de peintures, cycles de conférences...) au sein même de leur commune. Si elle n'empêche pas les 3/4 des répondants au questionnaire de sortir « souvent » de leur commune de résidence en semaine (la proportion tombant à $55 \%$ le week-end), cette inscription des pratiques quotidiennes à l'échelle du village participe de l'adhésion revendiquée à un mode de vie (assimilé $\mathrm{au}$ « rural »), qui consiste à fréquenter la nature à des fins récréatives mais aussi à investir volontairement les territoires les plus proches du logement qui contribuent à la valorisation de celui-ci (d'autant qu'ils accueillent les activités elles-mêmes valorisantes de la sociabilité et des loisirs).

L'analyse révèle également l'importance des échanges « de secteur », inscrits dans un rayon de dix à quinze kilomètres autour du domicile et correspondant à environ dix minutes de déplacement motorisé. Maillé de pôles de proximité, ce secteur est un archipel de lieux fréquentés « à la carte », investi sur le mode de l'idéal des réseaux et de ses valeurs d' " ubiquité, instantanéité, immédiateté de relations toujours permises mais choisies dans le temps et dans l'espace »(Dupuy, 1995). On est, en termes de représentations, dans « le tout proche » et le facile d'accès. Dans ce périmètre, les habitants accèdent à un ensemble varié de ressources, en premier lieu pour les activités d'achats et - toujours - de loisirs. Par le biais des associations, la quasi-totalité des ménages rencontrés pratiquent avec une régularité au moins hebdomadaire un loisir et/ou participent à des événements plus ponctuels, en général de récurrence annuelle mais suffisamment nombreux et variés pour qu'ils surviennent en cumulé tous les deux mois en moyenne. L'activité associative anime ainsi également le territoire de nombreux échanges intercommunaux. En effet, si l'aire de recrutement de beaucoup d'associations est principalement communale (renvoyant à l'une des échelles de l'ancrage), un certain nombre d'entre elles attirent à cette autre échelle du secteur et des dix minutes de trajet motorisé. C'est aussi dans ce rayon que s'exerce l'attraction des manifestations de la vie locale (carte 2). 
Carte 2 : La fréquentation préférentielle des communes proches pour le motif loisirs.

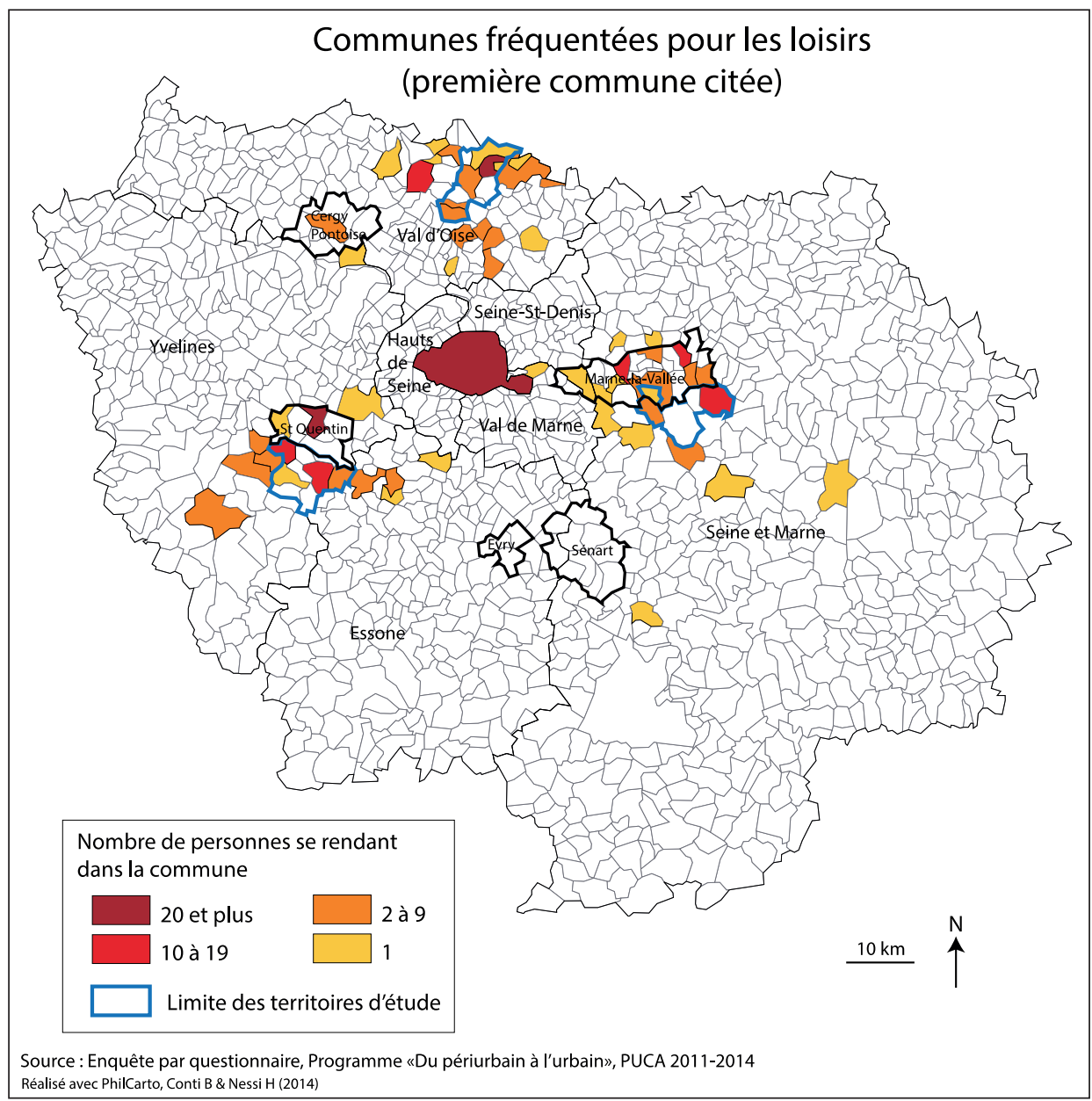

«Le 14 juillet, par exemple, on le fait à Pontcarré, mais on peut aussi bien le faire à Roissy-en-Brie ou aller à Ozoir, c'est le secteur géographique dans lequel on tourne. (...). On a des amis là-bas, et puis on aime bien bouger dans ces trois communes, quoi. » (Jérôme, Brie Boisée, Pontcarré, 51 ans, marié, agent RATP) «Ma femme fait de la marche à pied dans un groupe, c'est à $10 \mathrm{~km}$, puis elle va jouer aux cartes dans un groupe à Luzarches, elle joue ici aussi, mais le jeudi après-midi, elles se retrouvent, enfin elle a un groupe... Et puis, on a aussi une autre activité, on joue pas mal au scrabble dans un club à Chantilly. » (Jean-Michel, Carnelle - Pays de France, Seugy, 77 ans, marié, retraité)

«Il y a pas mal d'associations sur Montsoult, en fait. Montsoult, Maffliers, Baillet, c'est...ça fait partie de... on a une communauté... c'est vraiment... c'est le même code postal, il me semble même. » (Vincent, Carnelle - Pays de France, Baillet-enFrance, 49 ans, marié, informaticien) 
Dans sa double acception de « très proche » et de « secteur », le local est très investi par les pratiques de sociabilité et de loisirs qui se territorialisent autour de la double échelle de l'ancrage. Au sein du secteur de proximité, les personnes établissent aussi des préférences variables dans les lieux fréquentés, en fonction de la façon dont leurs réseaux sociaux les conduisent à territorialiser leurs activités et leur ont donné lecture des opportunités territoriales (cf. 2.3). Cette possibilité de moduler les fréquentations (sociales et territoriales) en associant le très proche et le plus lointain est un ressort important de l'aspiration à la proximité que manifestent les ménages périurbains. Elle régule aussi les mobilités quotidiennes générées par d'autres motifs.

\subsection{Une aspiration à la proximité qui ne se réduit pas à la métrique des kilomètres}

La reconstitution des schémas de déplacements permet bien de saisir un rétrécissement des bassins de vie pour les motifs d'achats et de loisirs, y compris culturels. Le renforcement du maillage des ressources de secteur ${ }^{24}$ est en effet l'occasion de mettre en œuvre des stratégies de proximité.

" [Depuis l'ouverture du Casino de Ferrières-en-Brie] Je ne vais plus jamais au centre commercial de Noisy-le-Grand. » (Josiane, Brie Boisée, Ferrières-en-Brie, 50 ans, célibataire, Institutrice)

Cette évolution des territoires fréquentés ne procède pas de la seule volonté d'économiser les kilomètres parcourus, d'autant que l'exploitation accrue de ces ressources relativement proches mais peu polarisées n'est pas favorable à une rationalisation effective de la mobilité. Elle correspond avant tout à la revendication par les ménages de comportements qui sont pour eux constitutifs de leur relation au territoire habité.

Ainsi l'évolution du tissu commercial a accompagné l'affirmation d'un mode de consommation caractérisé par le recours aux grandes et moyennes surfaces alimentaires ou spécialisées plutôt qu'aux très grands centres commerciaux, ainsi que par une part significative d'achats effectués dans les petits commerces (Motte-Baumvol, 2007 ; Bouleau et Mettetal, 2014). Le renforcement de cette maille commerciale intermédiaire en termes de surface comme de distance rencontre les aspirations des résidents à des modèles de consommation " à taille humaine ». Une nette dévalorisation affecte ainsi parallèlement hypermobilité et hyperconsommation. L'aspiration à la proximité a ici pour moteur l'économie de temps, moins en réduisant les kilomètres parcourus qu'en évitant la fréquentation chronophage de structures surdimensionnées. Elle relève aussi de l'adoption de modèles comportementaux valorisant, au travers de l'investissement dans la proximité de secteur, une frugalité que les ménages rencontrés assimilent au mode de vie rural et qui s'oppose non seulement à l'avatar du grand centre commercial périphérique mais aussi à un certain "superflu » urbain : les enquêtés déclarent ainsi avoir insensiblement abandonné le « lèche-vitrines » qu'ils pratiquaient en ville. Le rejet des complexes associant commerces et loisirs renvoie aussi à cette norme comportementale

\footnotetext{
${ }^{24}$ Les enquêtés mentionnent notamment l'ouverture, postérieure à leur arrivée, de piscines, cinémas et théâtres (particulièrement sur les terrains de la Brie boisée et de la Haute Vallée de Chevreuse qui bénéficient de la proximité des villes nouvelles) et de nombreuses surfaces alimentaires ou spécialisées à l'échelle du secteur. Cette évolution du tissu commercial caractérise la couronne distante de 20 à 40 km de Paris (Motte-Baumvol, 2007).
} 
qui impose de s'en tenir au «nécessaire » : ne pas assimiler les deux motifs, c'est refuser de prendre la consommation pour du loisir, et inversement.

Autre aspiration : les ménages revendiquent une forme d'itinérance dans leur pratique du territoire, expression valorisante de la variété des ressources à disposition et du refus des emplois du temps réglés (qui impacte également les modalités de relation à autrui, cf. partie 2). Dans ce rayon des dix minutes en voiture, ils modulent à volonté les caractéristiques des espaces fréquentés, alternant ainsi différents supermarchés mais aussi différents espaces verts ${ }^{25}$, ou encore différents événements festifs. La voiture permet aux individus de composer, à l'échelle du secteur, des programmes d'activités flexibles, finalement moins formatés par les impératifs de gestion de la distance et du temps que par le désir de faire varier les lieux et les temporalités, sans programmer, sans chaîner, sans régularité. Ainsi, le schéma de la «virée » hebdomadaire à l'hypermarché est loin d'être la règle sur nos terrains et la fréquentation des lieux d'achats même ordinaires est peu ritualisée.

«Les courses, on les fait un peu partout ... On n'a pas de lieu attitré. » (Jérôme, Brie Boisée, Pontcarré, 51 ans, marié, agent RATP)

Dans cet univers de choix, le passage par le domicile structure l'organisation des activités et des mobilités : il est le corollaire ritualisé du refus des programmations de long terme. Pour partie discursif, ce refus nous semble correspondre aussi à des pratiques effectives qu'on ne peut pas ou plus caractériser, contrairement aux analyses portant sur d'autres terrains périurbains, par un enchaînement marqué et programmé des motifs et des parcours (Dodier, 2007a). Elles renvoient à des modifications objectives des rythmes sociaux (affectant globalement la sphère productive du travail ou directement liées à l'installation périurbaine) et à l'avènement du « temps résidentiel » qui permet d'amorcer le processus d'ancrage.

"Je n'ai pas de rythme. Je me lève le matin, mais ma semaine n'est pas rythmée par des horaires, par des choses à faire, en arrêtant ma vie professionnelle, je ne voulais surtout pas ça. » (Carole, Haute Vallée de Chevreuse, Dampierre, 45 ans, mariée, décoratrice d'intérieur)

\subsection{La difficile appréhension du bassin d'ancrage par la puissance publique}

Si le recours à la proximité dans sa double acception de «très local » et « de secteur » est croissant, la combinaison entre les deux échelles de la proximité est loin d'être clairement hiérarchique. Elle évolue au gré du tissage des liens sociaux, de l'apprentissage des potentialités de la proximité et de l'évolution de l'offre du territoire. C'est alors tout un système d'échanges croisés qui se déploie entre des polarités locales offrant des caractéristiques variées (ici un supermarché bien accessible, là un autre avec des prix attractifs sur tel produit, ici un boucher réputé, etc.). Les flux définissent un réseau de lieux dont l'agencement reste à préciser, mais dont on peut faire l'hypothèse qu'il échappe par bien des aspects au modèle christallérien. Par exemple, les habitants de Coignières, commune bien équipée dont l'hypermarché rayonne sur la Haute vallée de Chevreuse, fréquentent

\footnotetext{
${ }^{25}$ Recherchant ici un paysage avec un point d'eau, là un lieu de balade avec un village pittoresque, ici une aire aménagée en « parc »...
} 
aussi les rues commerçantes du Mesnil-Saint-Denis. Les festivités locales, qui valent chacune pour leur(s) spécificité(s), sont aussi mises régulièrement en concurrence à l'échelle du secteur. De façon générale, s'il existe des arbitrages entre distance et niveau d'équipement, ce sont les modalités d'engagement dans le lien social qui permettent de comprendre l'établissement changeant des préférences et la façon dont se construit le bassin de vie des résidents du périurbain.

Le discours des élus, s’il confirme cette forme d'attractivité généralisée des lieux au sein d'un bassin d'ancrage tissé par les liens sociaux, l'assimile à du dysfonctionnement : les effets de concurrence territoriale se cumulent aux désordres de la circulation et au fait que le bassin d'ancrage que chaque individu construit à partir de l'épicentre de son domicile ne correspond pas aux périmètres institutionnels de l'intercommunalité.

«La fête de la musique, on a une excellente programmation, mais on a un problème de fréquentation. C'est très variable selon les années, parce que les gens tournent : un coup, ils viennent ici, un coup ils vont dans une commune à côté, parfois dans la même soirée! » (Entretien élu Haute Vallée de Chevreuse)

"[Le repas de Noël], on a arrêté parce qu'on était en déficit, et on s'est rendu compte qu'il y avait de moins en moins de monde de Seugy qui venait, c'était des gens de Viarmes, Chaumontel, Luzarches qui venaient, donc finalement on a pris la décision d'arrêter ce type de... ces animations; notre objectif n'était pas d'animer les villes environnantes, c'était d'animer un peu Seugy, donc on a cessé. » (Entretien élu Carnelle - Pays de France)

De fait, les initiatives d'animation et de promotion intercommunales du territoire sont rarissimes, la seule action dans le domaine consistant à afficher le calendrier des différentes festivités sur le site internet de la communauté de communes.

Enfin, il est très délicat pour la puissance publique de réguler et/ou de soutenir les créations auto-organisées de ressources locales ${ }^{26}$. L'élargissement du cercle des participants, la monétarisation et/ou la standardisation-systématisation du service sont perçus comme une distorsion grave de l'esprit initial. Ces pratiques sont souvent vouées à rester confidentielles et informelles. Une initiative habitante visant à «normer » la pratique spontanée de l'auto-stop en distribuant un macaron aux automobilistes résidents (assurant l'auto-stoppeur qu'il a affaire à quelqu'un « du cru») est ainsi restée lettre morte; et on ne compte sur nos terrains qu'une association à avoir adhéré au Système Local d'Entraide ${ }^{27}$. Malgré la vigueur du tissu associatif, les voies de l'économie collaborative restent à formaliser pour structurer des bassins d'ancrage qui fonctionnent de manière spontanée.

\section{Conclusion}

Qu'il soit ou non l'espace habité tout au long d'une vie, le périurbain génère un attachement qui s'hérite et se transmet. C'est un espace d'enracinement résidentiel mais aussi d'ancrage, animé de liens réguliers à des lieux qui dessinent un périmètre d'une grosse dizaine de kilomètres autour du domicile. Enracinement résidentiel et ancrage se ren-

${ }^{26}$ Sauf à en prendre intégralement la charge, comme dans le cas de la création à Seugy d'une cantine scolaire associative, reprise depuis par la mairie.

${ }^{27}$ Le SEL vise à faciliter les échanges en donnant une valeur comptabilisable au temps investi. 
forcent mutuellement : la découverte des ressources locales (matérielles et sociales) se fait à la faveur d'un projet d'installation inscrit dans la durée, qu'elle consolide en retour en créant de l'attachement.

Si l'automobile est un adaptateur territorial (Dupuy, 1995), les liens sociaux et le territoire, une fois ses opportunités décodées, sont des adaptateurs de mobilité. Ils rendent possible l'inscription de la vie quotidienne dans des périmètres de proximité et influencent l'agencement géographique des lieux fréquentés au sein du bassin d'ancrage. Bien que limitées au regard de la masse de l'autosolisme, les formes de gestion communautaire de la mobilité existent dans le périurbain. Il en va de même du lien social, même si les modalités de sa construction relèvent aussi de la construction d'un « entre-soi » (Donzelot, 1999). Les dynamiques associatives sont bien le moteur et la manifestation d'interactions multiples. C'est à partir d'elles que se construisent la connaissance et la maîtrise du territoire. Leur vigueur signale la volonté des résidents périurbains d'investir non seulement dans un logement mais aussi dans l'espace qui l'environne pour profiter de ses ressources. Dans cette démarche, lieux et groupes constituent des repères indispensables, aussi bien pour les personnes âgées ayant passé leur existence sur place que pour les «nouveaux » arrivants, dont l'identification progressive au territoire passe pour ainsi dire systématiquement par les relations nouées au sein d'une association.

Profiter des ressources locales ne signifie pas pour autant «s'assimiler » de manière figée à cet environnement. Cette liberté est revendiquée dans la réversibilité de l'engagement associatif comme dans le mouvement, qui s'organise dans le refus de la routine immuable, dans la multiplicité des lieux fréquentés, essayés, parfois abandonnés puis retrouvés au rythme décrété changeant des contraintes et des envies. L'ancrage n'étouffe pas « la ville au choix ». Le résident périurbain occupe, lui aussi, « un espace diversifié, multiple (...), [il] est nomade, multilocal, ubiquitaire » (Baudin, Bonnin, 2009). Mais en s'inscrivant dans une localité et dans une « société des voisins », il éprouve sa capacité d'interprétation et d'action pour reconstruire « sans fin ces contextes mêmes » (Haumont, 2005). Pour ce faire, il mobilise conjointement savoir-faire automobile et savoir-faire territorial, qu'on peut définir transversalement comme la capacité à utiliser des réseaux pour développer une lecture stratégique et personnelle d'un contexte territorial et infrastructurel largement modelé par des forces exogènes.

Il est tout-à-fait hors de propos d'affirmer que les modes de co-habitation analysés ici sont spécifiquement périurbains, ou qu'ils concernent tous les espaces périurbains compte tenu des caractéristiques sociales des terrains enquêtés ${ }^{28}$. Mais il reste que le processus d'ancrage, loin de se limiter à un enracinement résidentiel, révèle l'établissement progressif de liens qui «font avec» l'espace et ses caractéristiques, pallient ses insuffisances par l'autoorganisation et se jouent de ses limites pour « faire territoire » (Baudin, Bonnin, 2009).

${ }^{28}$ La pratique du village périurbain, qu'il s'agisse d'en fréquenter les commerces, les associations ou les manifestations festives, serait notamment moins développée chez les classes moyennes et populaires (Dodier 2007a). 


\section{Bibliographie}

Aguilera A., Nessi H., Sajous P., Thébert M. (resp.), Bonin O., Conti B., Coralli M., Le Néchet F., Proulhac L., Stransky V. et Terral L., 2014. Dynamiques du peuplement, des formes urbaines et des mobilités dans les territoires de la périurbanisation. Quels enseignements au regard des enjeux du développement durable? Rapport final pour le PUCA.

Aragau C., Berger M. et Rougé L., 2011. Du périurbain aux périurbains. Diversification sociale et générationnelle dans l'ouest francilien. Pouvoirs locaux 94, 58-64.

Aragau C. et Morel-Brochet A., 2010. Partir ou rester : l'ancrage résidentiel périurbain à l'épreuve du vieillissement In Membrado M., Rouyer A. (dir.), Habiter et vieillir, Érès, Toulouse, 105-120.

Authier J-Y., Bidet J., Collet A., Gilbert P. et Steinmetz H., 2010. État des lieux sur les trajectoires résidentielles. Rapport de recherche pour le PUCA.

Baudin G. et Bonnin P. (dir.), 2009. Faire territoire. Éditions Recherches, Paris.

Berger M., Aragau C. et Rougé L., 2014. Vers une maturité des territoires périurbains ? Développement des mobilités de proximité et renforcement de l'ancrage dans l'ouest francilien. EchoGéo [en ligne], 27, URL : http://echogeo.revues.org/13683

Bonnin-Oliveira S., Berger M., Aragau C., Rougé L., Desbordes F. et al., 2014. Les « pôles secondaires » dans la réorganisation des mobilités : maturité et durabilité des espaces périurbains ? Vivre en ville hors des villes. Synthèse de programme de recherche, MELT/MEDDE/PUCA, 29-41.

Bouleau M., Mettetal L., 2014. La mobilité dans le périurbain : désir d'ancrage et nouvelles proximités. Note rapide 646, Institut d'Aménagement et d'Urbanisme - Ile-de-France, mars 2014.

Breviglieri M., 2009. Le souci de propriété. Vie privée et déclin du militantisme dans un squat genevois In Pattaroni L., Kaufmann V. et Rabinovich A., Habitat en devenir. Enjeux territoriaux, politiques et sociaux du logement en Suisse, PPUR, Lausanne.

Cailly L., 2008. Existe-t-il un mode d'habiter spécifiquement périurbain ? L'exemple de l'aire urbaine d'une ville française (Tours). Espaces Temps.net, Travaux, 13/05/2008, http://www.espacestemps.net/articles/ mode-habiter-periurbain

Cailly L., 2014. Les habitants du périurbain tourangeau à l'épreuve d'un changement de modèle : vers une recomposition des modes d'habiter ? L'exemple des habitants de la ZAC des Terrasses de Bodets à Montlouis-sur-Loire. Norois 231, 75-90.

Cailly L. et Dodier R., 2007. La diversité des modes d'habiter périurbains dans les villes intermédiaires. Norois 205, 67-80.

Charmes, E., 2005. Entre ouverture et fermeture : les rapports à autrui dans les tissus périurbains In Haumont, B. et Morel, A., La société des voisins. Partager un habitat collectif. Maison des sciences de 1'Homme, 109-121.

Charmes E., 2008. Les périurbains sont-ils anti-urbains ? Version auteur revue en novembre 2008 de l'article paru dans Les Annales de la recherche urbaine 102, 7-18.

Conti B., 2012. Dynamiques de peuplement et de mobilité du périurbain francilien : quels enseignements au regard de la durabilité urbaine ? Mémoire de Master, Université Paris Est.

Corneloup J., Bourdeau J., Bachimon P. et Bessy O., 2014. L'habitabilité récréative périurbaine. Sociétés 125, 47-58.

Cousin B., 2014. Entre-soi mais chacun chez soi. L'agrégation affinitaire des cadres parisiens dans les espaces refondés. Actes de la recherche en sciences sociales 204, 88-101.

De Coninck F., 2010. Internet et la fracture des types de sociabilités In Massot H., Mobilités et modes de vie métropolitains, les intelligences du quotidien, Éd. L'œil d'or, Paris.

Di Méo G., 1996. Les territoires du quotidien. L’Harmattan, Paris.

Dodier R., 2007a. Les périurbains et la ville : entre individualisme et logiques collectives. Les Annales de la Recherche Urbaine, 31-39.

Dodier R., 2007b. Temporalités périurbaines : des navettes pendulaires à la fluidité et au conflit. Espace populations sociétés 2-3, 305-316. 
Dodier R. Cailly L., Gasnier A. et Madore F., 2012. Habiter les espaces périurbains. Presses Universitaires de Rennes, Rennes.

Donzelot J., 1999. La nouvelle question urbaine. Esprit 258, 87-114.

Dubost F., 1984. Côté jardins. Éditions du Scarabée, Paris.

Dupuy G., 1995. The Automobile System: a Territorial Adapter. Flux 21, 21-36.

Fortin A. et Després C., 2008. Le juste milieu : représentations de l'espace des résidents du pérurbain de l'agglomération de Québec. Cahiers de géographie du Québec 52, 153-174.

Fourny M-C., Cailly L. et Dodier R., 2012. La proximité, une ressource territoriale de la mobilité périurbaine. ASRDLF, $49^{\mathrm{e}}$ colloque international, 9 au 11 juillet, Belfort, France.

Girard V., 2014. Des classes populaires (encore) mobilisées ? Sociabilité et engagements municipaux dans une commune périurbaine. Espaces et Sociétés 156-157, 109-124.

Granovetter M., 1976. The Strength of Weak Ties. American Journal of Sociology 78 (6), 1360-1380.

Haumont B., 2005. Préface : Entre public et privé : des espaces et des lieux toujours en chantier In Haumont B. et Morel A., La société des voisins. Éditions de la Maison des sciences de l'Homme, Paris.

Hilal M., Sencébé Y., 2002. Mobilité quotidienne et urbanité suburbaine. Espaces et Société, 108/109, 2002, 133-153.

Mathieu N., 2012. Les modes d'habiter. À l'origine d'un concept. In Morel-Brochet A., Ortar N., La fabrique des modes d'habiter. Hommes, lieux et milieux de vie. L'Harmattan, Paris, 35-53.

Motte-Baumvol B., 2007. Les populations périurbaines face à l'automobile en grande couronne francilienne. Norois 205, 53-66.

Nessi H., 2012. Influence du contexte urbain et du rapport au cadre de vie sur la mobilité de loisir en Ile-de-France et à Rome. Thèse de doctorat Université Paris Est, sous la dir. de Coutard O., soutenue le 30 novembre 2012

Pattaroni L., Kaufmann V. et Rabonovitch A. (dir.), 2009. Habitat en devenir : enjeux territoriaux, politiques et sociaux du logement en Suisse. PPUR, Lausanne.

Pinson D. et Thomann S., 2002. La maison en ses territoires. De la villa à la ville diffuse. L'Harmattan, Paris.

Pradel B., Cailly L., Fourny M.-C., Chardonnel S., Dodier R. et Louargant S., 2014. Relations sociales et solidarités collectives dans les déplacements périurbains : vers une identité de mouvement. Recherche Transport Sécurité 2-3, 125-141.

Préteceille E., 2003.La division sociale de l'espace francilien. Typologie socio-professionnelle 1999 et transformations de l'espace résidentiel 199099. Paris : Observatoire sociologique du changement (FNSP/CNRS).

Rougé L., 2012. Retour dans les espaces de la « captivité périurbaine » : diversification des parcours de vie et affirmation de logiques d'autonomisation. Sud Ouest Européen 31, 43-53.

Sajous, P., 2004. Habiter en périurbain et usages de la voiture. Revue ESO Travaux et documents 21.

Sencébé Y. et Hilal M., 2012. Quelle durabilité « sociale » révèlent les pratiques de sociabilité et les pratiques culturelles et de loisir ? In Hilal M. (resp.), Cinquante ans de mobilités résidentielles et quotidiennes : quelles formes territoriales pour un mode de vie durable? Rapport de recherche pour le PUCA.

Stock M., 2001. Mobilités géographiques et pratiques des lieux. Étude théorico-empirique à travers deux lieux touristiques anciennement constitués : Brighton \& Hove (Royaume-Uni) et Garmisch-Partenkirchen (Allemagne). Thèse de géographie, Université de Paris 7. 